\title{
Hedging of covered options with linear market impact and gamma constraint
}

\author{
B. Bouchard*† G. Loeper ${ }^{\ddagger}$ and Y. Zou*
}

December 23, 2015

\begin{abstract}
Within a financial model with linear price impact, we study the problem of hedging a covered European option under gamma constraint. Using stochastic target and partial differential equation smoothing techniques, we prove that the super-replication price is the viscosity solution of a fully non-linear parabolic equation. As a by-product, we show how $\varepsilon$-optimal strategies can be constructed. Finally, a numerical resolution scheme is proposed.
\end{abstract}

Keywords: Hedging, Price impact, Stochastic target.

AMS 2010 Subject Classification: 91G20; 93E20; 49L20

\section{Introduction}

Inspired by [1, 18, the authors in [4] considered a financial market with permanent price impact, in which the impact function behaves as a linear function (around the origin) in the number of bought stocks. This class of models is dedicated to the pricing and hedging of derivatives in situations where the notional of the product hedged is such that the delta-hedging is non-negligible compared to the average daily volume traded on the underlying asset. Hence, the delta-hedging strategy will

*Paris-Dauphine University, PSL Research University, CEREMADE, UMR 7534, 75775 Paris cedex 16, France.

${ }^{\dagger}$ Research supported by ANR Liquirisk

${ }^{\ddagger}$ Monash University, School of Mathematical Sciences, Victoria 3800 Australia. 
have an impact on the price dynamics, and will also incur liquidity costs. The linear impact models studied in [1, 4, 18] incorporate both the effects into the pricing and hedging of the derivative, while maintaining the completeness of the market (up to a certain extent) and eventually leading to exact replication strategies. As in perfect market models, this approach can provide an approximation of the real market conditions that can be used by practitioners to help them in the design of a suitable hedge in a systematic way, without having to rely on an ad hoc risk criterion.

In [4], the authors considered the hedging of a cash-settled European option: at inception the option seller has to build the initial delta-hedge, and conversely at maturity the hedge must be liquidated to settle the final claim in cash. It is shown therein that the price function of the optimal super-replicating strategy no longer solves a linear parabolic equation, as in the classical case, rather a quasi-linear one. Moreover, the hedging strategy consists in following a modified delta-hedging rule where the delta is computed at the "unperturbed" value of the underlying, i.e., the one the underlying would have if the trader's position were liquidated immediately.

The approach and the results obtained in [4] thus differ substantially from [1, 18]. Indeed, while in [1, 18] the impact model considered is the same, the control problem is different in the sense that it is applied to the hedging of covered options. This refers to situations where the buyer of the option delivers at inception the required initial delta position, and accepts a mix of stocks (at their current market price) and cash as payment of the final claim, which eliminates the cost incurred by the initial and final hedge. Quite surprisingly, this is not a genuine approximation of the problem studied in [4]. The question of the initial and final hedge is fundamental, to the point that the structure of the pricing question is completely different: in [4] the equation is quasi-linear, while it is fully non-linear in [1, 18].

As opposed to [4], authors in [1, 18] use a verification argument to build an exact replication strategy. Due to the special form of the non-linearity, the equation is ill-posed when the solution does not satisfy a gamma-type constraint. The aim of the current paper is to provide a direct characterization via stochastic target techniques, and to incorporate right from the beginning a gamma constraint on the hedging strategy.

The super-solution property can be proved by (essentially) following the arguments of [8]. The sub-solution characterization is much more difficult to obtain. Actually, we could not prove the required geometric dynamic programming principle, unlike [8], because of the strong interaction between the hedging strategy and the underlying price process due to the market impact. Instead, we use the smoothing technique developed in [5]. We construct a sequence of smooth super-solutions which, by a verification argument, provide upper-bounds for the super-hedging 
price. As they converge to a solution of the targeted pricing equation, a comparison principle argument implies that their limit is the super-hedging price. As a by-product, this construction provides explicit $\varepsilon$-optimal hedging strategies. We also provide a comparison principle and a numerical resolution scheme. For simplicity, we first consider a model that has only permanent price impact, we explain in Section 3 why adding a resilience effect does not affect our analysis. Note that this is because the resilience effect we consider here has no quadratic variation, as opposed to [1], in which the resilience can destroy the parabolicity of the equation, and renders the exact replication non optimal.

We close this introduction by pointing out some related references. [6] incorporates liquidity costs but no price impact, the price curve is not affected by the trading strategy. It can be modified by adding restrictions on admissible strategies as in [7] and [24]. This leads to a modified pricing equation, which exhibits a quadratic term in the second order derivative of the solution, and renders the pricing equation fully non-linear, and even not unconditionally parabolic. Other articles focus on the derivation of the price dynamics through clearing condition, see e.g., [12], 22], 21] in which the supply and demand curves arise from "reference" and "program" traders (i.e., option hedgers); leading to a modified price dynamics, but without taking into account the liquidity costs, see also [17]. Finally, the series of papers [23], 8], 24] addresses the liquidity issue indirectly by imposing bounds on the "gamma" of admissible trading strategies, no liquidity cost or price impact are modeled explicitly.

General notations. Throughout this paper, $\Omega$ is the canonical space of continuous functions on $\mathbb{R}_{+}$starting at $0, \mathbb{P}$ is the Wiener measure, $W$ is the canonical process, and $\mathbb{F}=\left(\mathcal{F}_{t}\right)_{t \geq 0}$ is the augmentation of its raw filtration $\mathbb{F}^{\circ}=\left(\mathcal{F}_{t}^{\circ}\right)_{t \geq 0}$. All random variables are defined on $\left(\Omega, \mathcal{F}_{\infty}, \mathbb{P}\right)$. We denote by $|x|$ the Euclidean norm of $x \in \mathbb{R}^{n}$, the integer $n \geq 1$ is given by the context. Unless otherwise specified, inequalities involving random variables are taken in the $\mathbb{P}-$ a.s. sense. We use the convention $x / 0=\operatorname{sign}(x) \times \infty$ with $\operatorname{sign}(0)=+$.

\section{Model and hedging problem}

This section is dedicated to the derivation of the dynamics and the description of the gamma constraint. We also explain in detail how the pricing equation can be obtained and state our main result. 


\subsection{Impact rule and discrete time trading dynamics}

We consider the framework studied in [4]. Namely, the impact of a strategy on the price process is modeled by an impact function $f$ : the price variation due to buying a (infinitesimal) number $\delta \in \mathbb{R}$ of shares is $\delta f(x)$, given that the price of the asset is $x$ before the trade. The cost of buying the additional $\delta$ units is

$$
\delta x+\frac{1}{2} \delta^{2} f(x)=\delta \int_{0}^{\delta} \frac{1}{\delta}(x+\iota f(x)) d \iota,
$$

in which

$$
\int_{0}^{\delta} \frac{1}{\delta}(x+\iota f(x)) d \iota
$$

can be interpreted as the average cost for each additional unit.

Between two trading instances $\tau_{1}, \tau_{2}$ with $\tau_{1} \leq \tau_{2}$, the dynamics of the stock is given by the strong solution of the stochastic differential equation

$$
d X_{t}=\mu\left(X_{t}\right) d t+\sigma\left(X_{t}\right) d W_{t}
$$

Throughout this paper, we assume that

$$
\begin{gathered}
f \in C_{b}^{2} \text { and } \inf f>0, \\
(\mu, \sigma) \text { is Lipschitz and bounded, } \inf \sigma>0 .
\end{gathered}
$$

As in 4, the number of shares the trader would like to hold is given by a continuous Itô process $Y$ of the form

$$
Y=Y_{0}+\int_{0}^{\cdot} b_{s} d s+\int_{0}^{\cdot} a_{s} d W_{s}
$$

We say ${ }^{1}$ that $(a, b)$ belongs to $\mathcal{A}_{k}^{\circ}$ if $(a, b)$ is continuous, $\mathbb{F}$-adapted,

$$
a=a_{0}+\int_{0}^{\cdot} \beta_{s} d s+\int_{0}^{\cdot} \alpha_{s} d W_{s}
$$

where $(\alpha, \beta)$ is continuous, $\mathbb{F}$-adapted, and $\zeta:=(a, b, \alpha, \beta)$ is essentially bounded by $k$ and such that

$$
\mathbb{E}\left[\sup \left\{\left|\zeta_{s^{\prime}}-\zeta_{s}\right|, t \leq s \leq s^{\prime} \leq s+\delta \leq T\right\} \mid \mathcal{F}_{t}^{\circ}\right] \leq k \delta
$$

for all $0 \leq \delta \leq 1$ and $t \in[0, T-\delta]$.

\footnotetext{
${ }^{1}$ In [4], $(a, b)$ is only required to be progressively measurable and essentially bounded. The additional restrictions imposed here will be necessary for our results in Section 2.2 .
} 
We then define

$$
\mathcal{A}^{\circ}:=\cup_{k} \mathcal{A}_{k}^{\circ} .
$$

To derive the continuous time dynamics, we first consider a discrete time setting and then pass to the limit. In the discrete time setting, the position is re-balanced only at times

$$
t_{i}^{n}:=i T / n, i=0, \ldots, n, n \geq 1 .
$$

In other words, the trader keeps the position $Y_{t_{i}^{n}}$ in stocks over each time interval $\left[t_{i}^{n}, t_{i+1}^{n}\right)$. Hence, his position in stocks at $t$ is

$$
Y_{t}^{n}:=\sum_{i=0}^{n-1} Y_{t_{i}^{n}} \mathbf{1}_{\left\{t_{i}^{n} \leq t<t_{i+1}^{n}\right\}}+Y_{T} \mathbf{1}_{\{t=T\}}
$$

and the number of shares purchased at $t_{i+1}^{n}$ is

$$
\delta_{t_{i+1}^{n}}^{n}:=Y_{t_{i+1}^{n}}-Y_{t_{i}^{n}}
$$

Given our impact rule, the corresponding dynamics for the stock price process is

$$
X^{n}=X_{0}+\int_{0} \mu\left(X_{s}^{n}\right) d s+\int_{0}^{.} \sigma\left(X_{s}^{n}\right) d W_{s}+\sum_{i=1}^{n} \mathbf{1}_{\left[t_{i}^{n}, T\right]} \delta_{t_{i}^{n}}^{n} f\left(X_{t_{i}^{n}-}^{n}\right),
$$

in which $X_{0}$ is a constant.

The portfolio process is described as the sum $V^{n}$ of the amount of cash held and the potential wealth $Y^{n} X^{n}$ associated to the position in stocks:

$$
V^{n}=\text { cash position }+Y^{n} X^{n} \text {. }
$$

It does not correspond to the liquidation value of the portfolio, except when $Y^{n}=0$. This is due to the fact that the liquidation of $Y^{n}$ stocks does not generate a gain equal to $Y^{n} X^{n}$, because of the price impact. However, one can infer the exact composition in cash and stocks of the portfolio from the knowledge of the couple $\left(V^{n}, Y^{n}\right)$.

Throughout this paper, we assume that the risk-free interest rate is zero (for ease of notations). Then,

$$
V^{n}=V_{0}+\int_{0}^{\cdot} Y_{s-}^{n} d X_{s}^{n}+\sum_{i=1}^{n} \mathbf{1}_{\left[t_{i}^{n}, T\right]} \frac{1}{2}\left(\delta_{t_{i}^{n}}^{n}\right)^{2} f\left(X_{t_{i}^{n}-}^{n}\right) .
$$

This wealth equation is derived as in [4] following elementary calculations. The last term of the right-hand side comes from the fact that, at time $t_{i}^{n}, \delta_{t_{i}^{n}}^{n}$ shares are bought at the average execution price $X_{t_{i}^{n}-}^{n}+\frac{1}{2} \delta_{t_{i}^{n}}^{n} f\left(X_{t_{i}^{n}-}^{n}\right)$, and the stock's price ends at $X_{t_{i}^{n}-}^{n}+\delta_{t_{i}^{n}}^{n} f\left(X_{t_{i}^{n}-}^{n}\right)$, whence the additional profit term. However, one can check that a profitable round trip trade can not be built, see [4, Remark 3]. 
Remark 1.1. Note that in this work we restrict ourselves to a permanent price impact, no resilience effect is modeled. We shall explain in Section 3 below why taking resilience into account does not affect our analysis. See in particular Proposition 3.1.

\subsection{Continuous time trading dynamics}

The continuous time trading dynamics is obtained by passing to the limit $n \rightarrow \infty$, i.e., by considering strategies with increasing frequency of rebalancement.

Proposition 1.2. [4, Proposition 1] Let $Z:=(X, Y, V)$ where $Y$ is defined as in (1.2) for some $(a, b) \in \mathcal{A}^{\circ}$, and $(X, V)$ solves

$$
\begin{aligned}
X & =X_{0}+\int_{0}^{\cdot} \sigma\left(X_{s}\right) d W_{s}+\int_{0}^{\cdot} f\left(X_{s}\right) d Y_{s}+\int_{0}\left(\mu\left(X_{s}\right)+a_{s}\left(\sigma f^{\prime}\right)\left(X_{s}\right)\right) d s \\
& =X_{0}+\int_{0} \sigma_{X}^{a_{s}}\left(X_{s}\right) d W_{s}+\int_{0} \mu_{X}^{a_{s}, b_{s}}\left(X_{s}\right) d s
\end{aligned}
$$

with

$$
\sigma_{X}^{a_{s}}:=\left(\sigma+a_{s} f\right), \mu_{X}^{a_{s}, b_{s}}:=\left(\mu+b_{s} f+a_{s} \sigma f^{\prime}\right)
$$

and

$$
V=V_{0}+\int_{0}^{\cdot} Y_{s} d X_{s}+\frac{1}{2} \int_{0}^{\cdot} a_{s}^{2} f\left(X_{s}\right) d s
$$

Let $Z^{n}:=\left(X^{n}, Y^{n}, V^{n}\right)$ be defined as in (1.4)-(1.3)-(1.5). Then, there exists a constant $C>0$ such that

$$
\sup _{[0, T]} \mathbb{E}\left[\left|Z^{n}-Z\right|^{2}\right] \leq C n^{-1}
$$

for all $n \geq 1$.

For the rest of the paper, we shall therefore consider (1.7)- $(1.6)$ for the dynamics of the portfolio and price processes.

Remark 1.3. As explained in [4], the previous analysis could be extended to a non-linear impact rule in the size of the order. To this end, we note that the continuous time trading dynamics described above would be the same for a more general impact rule $\delta \mapsto F(x, \delta)$ whenever it satisfies $F(x, 0)=\partial_{\delta \delta}^{2} F(x, 0)=0$ and $\partial_{\delta} F(x, 0)=f(x)$. For our analysis, we only need to consider the value and the slope of the impact function at the origin. 


\subsection{Hedging equation and gamma constraint}

Given $\phi=(y, a, b) \in \mathbb{R} \times \mathcal{A}^{\circ}$ and $(t, x, v) \in[0, T] \times \mathbb{R} \times \mathbb{R}$, we now write $\left(X^{t, x, \phi}\right.$, $\left.Y^{t, \phi}, V^{t, x, v, \phi}\right)$ for the solution of $(1.6)-(1.2)-(1.7)$ associated to the control $(a, b)$ with time-t initial condition $(x, y, v)$.

In this paper, we consider covered options, in the sense that the trader is given at the initial time $t$ the number of shares $Y_{t}=y$ required to launch his hedging strategy and can pay the option's payoff at $T$ in cash and stocks (evaluated at their time- $T$ value). Therefore, he does not exert any immediate impact at time $t$ nor $T$ due to the initial building or final liquidation of his position in stocks. Recalling that $V$ stands for the sum of the position in cash and the number of held shares multiplied by their price, the super-hedging price at time $t$ of the option with payoff $g\left(X_{T}^{t, x, \phi}\right)$ is defined as

$$
\mathrm{v}(t, x):=\inf \left\{v=c+y x:(c, y) \in \mathbb{R}^{2} \text { s.t. } \mathcal{G}(t, x, v, y) \neq \emptyset\right\},
$$

in which $\mathcal{G}(t, x, v, y)$ is the set of elements $(a, b) \in \mathcal{A}^{\circ}$ such that $\phi:=(y, a, b)$ satisfies

$$
V_{T}^{t, x, v, \phi} \geq g\left(X_{T}^{t, x, \phi}\right) .
$$

In order to understand what the associated partial differential equation is, let us first rewrite the dynamics of $Y$ in terms of $X$ :

$$
d Y_{t}^{t, \phi}=\gamma_{Y}^{a_{t}}\left(X_{t}^{t, x, \phi}\right) d X_{t}^{t, x, \phi}+\mu_{Y}^{a_{t}, b_{t}}\left(X_{t}^{t, x, \phi}\right) d t
$$

with

$$
\gamma_{Y}^{a}:=\frac{a}{\sigma+f a} \quad \text { and } \quad \mu_{Y}^{a, b}:=b-\gamma_{Y}^{a} \mu_{X}^{a, b} .
$$

Assuming that the hedging strategy consists in tracking the super-hedging price, as in classical complete market models, then one should have $V^{t, x, v, \phi}=\mathrm{v}\left(\cdot, X^{t, x, \phi}\right)$. If $\mathrm{v}$ is smooth, recalling (1.6)-(1.7) and applying Itô's lemma twice implies

$$
Y^{t, \phi}=\partial_{x} \mathrm{v}\left(\cdot, X^{t, x, \phi}\right), \gamma_{Y}^{a}\left(X^{t, x, \phi}\right)=\partial_{x x}^{2} \mathrm{v}\left(\cdot, X^{t, x, \phi}\right),
$$

and

$$
\frac{1}{2} a^{2} f\left(X^{t, x, \phi}\right)=\partial_{t} \mathrm{v}\left(\cdot, X^{t, x, \phi}\right)+\frac{1}{2}\left(\sigma_{X}^{a}\right)^{2}\left(X^{t, x, \phi}\right) \partial_{x x}^{2} \mathrm{v}\left(\cdot, X^{t, x, \phi}\right) .
$$

Then, the right-hand side of 1.9 combined with the definition of $\gamma_{Y}^{a}$ leads to

$$
a=\frac{\sigma \partial_{x x}^{2} \mathrm{v}\left(\cdot, X^{t, x, \phi}\right)}{1-f \partial_{x x}^{2} \mathrm{v}\left(\cdot, X^{t, x, \phi}\right)} \quad, \quad \sigma_{X}^{a}=\frac{\sigma}{1-f \partial_{x x}^{2} \mathrm{v}\left(\cdot, X^{t, x, \phi}\right)},
$$


and 1.10$)$ simplifies to

$$
\left[-\partial_{t} \mathrm{v}-\frac{1}{2} \frac{\sigma^{2}}{\left(1-f \partial_{x x}^{2} \mathrm{v}\right)} \partial_{x x}^{2} \mathrm{v}\right]\left(\cdot, X^{t, x, \phi}\right)=0 \quad \text { on }[t, T) .
$$

This is precisely the pricing equation obtained in [1, 18].

Equation (1.11) needs to be considered with some precautions due to the singularity at $f \partial_{x x}^{2} \mathrm{v}=1$. Hence, one needs to enforce that $1-f \partial_{x x}^{2} \mathrm{v}$ does not change sign. We choose to restrict the solutions to satisfy $1-f \partial_{x x}^{2} \mathrm{v}>0$, since having the opposite inequality would imply that $a$ does not have the same sign as $\partial_{x x}^{2} \mathrm{v}$, so that, having sold a convex payoff, one would sell when the stock goes up and buy when it goes down, a very counter-intuitive fact.

In the following, we impose that the constraint

$$
-k \leq \gamma_{Y}^{a}\left(X^{t, x, \phi}\right) \leq \bar{\gamma}\left(X^{t, x, \phi}\right) \text {, on }[t, T] \mathbb{P} \text { - a.e., }
$$

should hold for some $k \geq 0$, in which $\bar{\gamma}$ is a bounded continuous map satisfying

$$
\iota \leq \bar{\gamma} \leq 1 / f-\iota, \quad \text { for some } \iota>0 .
$$

We denote by $\mathcal{A}_{k, \bar{\gamma}}(t, x)$ the collection of elements $(a, b) \in \mathcal{A}_{k}^{\circ}$ such that 1.12 holds and define

$$
\mathcal{A}_{\bar{\gamma}}(t, x):=\cup_{k \geq 0} \mathcal{A}_{k, \bar{\gamma}}(t, x)
$$

Then, the equation (1.11) has to be modified to take this gamma constraint into account, leading naturally to

$$
F\left[\mathrm{v}_{\bar{\gamma}}\right]:=\min \left\{-\partial_{t} \mathrm{v}_{\bar{\gamma}}-\frac{1}{2} \frac{\sigma^{2}}{1-f \partial_{x x}^{2} \mathrm{v}_{\bar{\gamma}}} \partial_{x x}^{2} \mathrm{v}_{\bar{\gamma}}, \bar{\gamma}-\partial_{x x}^{2} \mathrm{v}_{\bar{\gamma}}\right\}=0 \quad \text { on }[0, T) \times \mathbb{R},
$$

in which $\mathrm{v}_{\bar{\gamma}}$ is defined as $\mathrm{v}$ but with

$$
\mathcal{G}_{\bar{\gamma}}(t, x, v, y):=\mathcal{G}(t, x, v, y) \cap \mathcal{A}_{\bar{\gamma}}(t, x)
$$

in place of $\mathcal{G}(t, x, v, y)$. More precisely,

$$
\mathrm{v}_{\bar{\gamma}}(t, x):=\inf \left\{v=c+y x:(c, y) \in \mathbb{R}^{2} \text { s.t. } \mathcal{G}_{\bar{\gamma}}(t, x, v, y) \neq \emptyset\right\} .
$$

As for the $T$-boundary condition, we know that $\mathrm{v}_{\bar{\gamma}}(T, \cdot)=g$ by definition. However, as usual, the constraint on the gamma in (1.14) should propagate up to the boundary and $g$ has to be replaced by its face-lifted version $\hat{g}$, defined as the smallest function above $g$ that is a viscosity super-solution of the equation 
$\bar{\gamma}-\partial_{x x}^{2} \varphi \geq 0$. It is obtained by considering any twice continuously differentiable function $\bar{\Gamma}$ such that $\partial_{x x}^{2} \bar{\Gamma}=\bar{\gamma}$, and then setting

$$
\hat{g}:=(g-\bar{\Gamma})^{\text {conc }}+\bar{\Gamma},
$$

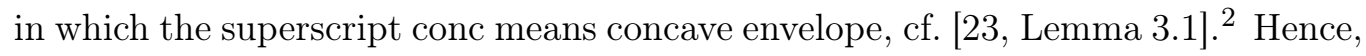
we expect that

$$
\mathrm{v}_{\bar{\gamma}}(T-, \cdot)=\hat{g} \text { on } \mathbb{R} .
$$

From now on, we assume that

$$
\hat{g} \text { is uniformly continuous, }
$$

$g$ is lower-semicontinuous, $g^{-}$is bounded and $g^{+}$has linear growth.

We are now in a position to state our main result. From now on

$$
\mathrm{v}_{\bar{\gamma}}(T, x) \text { stands for } \lim _{\substack{\left(t^{\prime}, x^{\prime}\right) \rightarrow(T, x) \\ t^{\prime}<T}} \mathrm{v}_{\bar{\gamma}}\left(t^{\prime}, x^{\prime}\right)
$$

whenever it is well defined.

Theorem 1.4. The value function $\mathrm{v}_{\bar{\gamma}}$ is continuous with linear growth. Moreover, $\mathrm{v}_{\bar{\gamma}}$ is the unique viscosity solution with linear growth of

$$
F[\varphi] \mathbf{1}_{[0, T)}+(\varphi-\hat{g}) \mathbf{1}_{\{T\}}=0 \text { on }[0, T] \times \mathbb{R} .
$$

We conclude this section with additional remarks.

Remark 1.5. Note that $\hat{g}$ can be uniformly continuous without $g$ being continuous. Take for instance $g(x)=\mathbf{1}_{\{x \geq K\}}$ with $K \in \mathbb{R}$, and consider the case where $\bar{\gamma}>0$ is a constant. Then, $\hat{g}(x)=\left[\mathbf{1}_{\left\{x \geq x_{o}\right.} \frac{\bar{\gamma}}{2}\left(x-x_{o}\right)^{2}\right] \wedge 1$ with $x_{o}:=K-(2 / \bar{\gamma})^{\frac{1}{2}}$.

Remark 1.6. The map $\hat{g}$ inherits the linear growth of $g$. Indeed, let $c_{0}, c_{1} \geq 0$ be constants such that $|g(x)| \leq w(x):=c_{0}+c_{1}|x|$. Since $\hat{g} \geq g$ by construction, we have $\hat{g}^{-} \leq w$. On the other hand, since $\bar{\gamma} \geq \iota>0$, by 1.13 , it follows from the arguments in [23, Lemma 3.1] that $\hat{g} \leq(w-\tilde{\Gamma})^{\text {conc }}+\tilde{\Gamma}$, in which $\tilde{\Gamma}(x)=\iota x^{2} / 2$. Now, one can easily check by direct computations that

$$
(w-\tilde{\Gamma})^{\mathrm{conc}}=(w-\tilde{\Gamma})\left(x_{o}\right) \mathbf{1}_{\left[-x_{o}, x_{o}\right]}+(w-\tilde{\Gamma}) \mathbf{1}_{\left[-x_{o}, x_{o}\right]^{c}}
$$

with $x_{o}:=c_{1} / \iota$. Hence, $(w-\tilde{\Gamma})^{\text {conc }}+\tilde{\Gamma}$ has the same linear growth as $w$.

\footnotetext{
${ }^{2}$ Obviously, adding an affine map to $\bar{\Gamma}$ does not change the definition of $\hat{g}$.
} 
Remark 1.7. As will appear in the rest of our analysis, one could very well introduce a time dependence in the impact function $f$ and in $\bar{\gamma}$. Another interesting question studied by the second author in [18] and [19] concerns the smoothness of the solution and how the constraint on $\partial_{x x}^{2} \mathrm{v}$ gets naturally enforced by the fast diffusion arising when $1-f \partial_{x x}^{2} \mathrm{v}$ is close to 0 .

Remark 1.8 (Existence of a smooth solution to the original partial differential equation). When the pricing equation (1.17) admits smooth solutions (cf. [18] and [19]) that allow to use the verification theorem, then one can construct exact replication strategies from the classical solution. By the comparison principle of Theorem 2.11 below, this shows that the value function is the classical solution of the pricing equation, and that the optimal strategy exists and is an exact replication strategy of the option with payoff function $\hat{g}$. We will explain in Remark 2.18 below how almost optimal super-hedging strategies can be constructed explicitly even when no smooth solution exists.

Remark 1.9 (Monotonicity in the impact function). Note that the map $\lambda \in \mathbb{R} \mapsto$ $\frac{\sigma^{2}(x) M}{1-\lambda M}$ is non-decreasing on $\{\lambda: \lambda M<1\}$, for all $(t, x, M) \in[0, T] \times \mathbb{R} \times \mathbb{R}$. Let us now write $\mathrm{v}_{\bar{\gamma}}$ as $\mathrm{v}_{\bar{\gamma}}^{f}$ to emphasize its dependence on $f$, and consider another impact function $\tilde{f}$ satisfying the same requirements as $f$. We denote by $\mathrm{v}_{\bar{\gamma}}^{\tilde{f}}$ the corresponding super-hedging price. Then, the above considerations combined with Theorem 1.4 and the comparison principle of Theorem 2.11 below imply that $\mathrm{v}_{\bar{\gamma}} \geq$

$\mathrm{v}_{\bar{\gamma}}^{f}$ whenever $\tilde{f} \geq f$ on $\mathbb{R}$. The same implies that $\mathrm{v} \frac{f}{\bar{\gamma}} \geq \mathrm{v}$ in which $\mathrm{v}$ solves the heat-type equation

$$
-\partial_{t} \varphi-\frac{1}{2} \sigma^{2} \partial_{x x}^{2} \varphi=0 \text { on }[0, T) \times \mathbb{R}
$$

with terminal condition $\varphi(T, \cdot)=g$ (recall that $\hat{g} \geq g$ ). See Section 4.2 for a numerical illustration of this fact.

\section{Viscosity solution characterization}

In this section, we provide the proof of Theorem 1.4. Our strategy is the following.

1. First, we adapt the partial differential equation smoothing technique used in [5] to provide a smooth supersolutions $\overline{\mathrm{v}}_{\bar{\gamma}}^{\epsilon, K, \delta}$ of 1.17 on $[\delta, T] \times \mathbb{R}$, with $\epsilon>0$, from which super-hedging strategies can be constructed by a standard verification argument. In particular, $\overline{\mathrm{v}}_{\bar{\gamma}}^{\epsilon, K, \delta} \geq \mathrm{v}_{\bar{\gamma}}$ on $[\delta, T] \times \mathbb{R}$. Moreover, this sequence has a uniform linear growth and converges to a viscosity solution $\overline{\mathrm{v}}_{\bar{\gamma}}$ of (1.17) as $\delta, \epsilon \rightarrow 0$ and $K \rightarrow \infty$. See Section 2.1. 
2. Second, we construct a lower bound $\mathrm{v}_{\bar{\gamma}}$ for $\mathrm{v}_{\bar{\gamma}}$ that is a supersolution of (1.17). It is obtained by considering a weak formulation of the super-hedging problem and following the arguments of [8, Section 5] based on one side of the geometric dynamic programming principle, see Section 2.2. It is shown that this function has linear growth as well.

3. We can then conclude by using the above and the comparison principle for (1.17) of Theorem 2.11 below: $\underline{\mathrm{v}}_{\bar{\gamma}} \geq \overline{\mathrm{v}}_{\bar{\gamma}}$ but $\underline{\mathrm{v}}_{\bar{\gamma}} \leq \mathrm{v}_{\bar{\gamma}} \leq \overline{\mathrm{v}}_{\bar{\gamma}}$ so that $\mathrm{v}_{\bar{\gamma}}=\overline{\mathrm{v}}_{\bar{\gamma}}=$ $\underline{\mathrm{v}}_{\bar{\gamma}}$ and $\mathrm{v}_{\bar{\gamma}}$ is a viscosity solution of (1.17), and has linear growth.

4. Our comparison principle, Theorem 2.11 below, allows us to conclude that $\mathrm{v}_{\bar{\gamma}}$ is the unique solution of (1.17) with linear growth.

As already mentioned in the introduction, unlike [8], we could not prove the required geometric dynamic programming principle that should directly lead to a subsolution property (thus avoiding to use the smoothing technique mentioned in 1. above). This is due to the strong interaction between the hedging strategy and the underlying price process through the market impact. Such a feedback effect is not present in [8].

\subsection{A sequence of smooth supersolutions}

We first construct a sequence of smooth supersolutions $\overline{\mathrm{v}}_{\bar{\gamma}}^{\epsilon, K, \delta}$ of 1.17) which appears to be an upper bound for the super-hedging price $\mathrm{v}_{\bar{\gamma}}$, by a simple verification argument. For this, we adapt the methodology introduced in [5]: we first construct a viscosity solution of a version of (1.17) with shaken coefficients (in the terminology of [15]) and then smooth it out with a kernel. The main difficulty here is that our terminal condition $\hat{g}$ is unbounded, unlike [5]. This requires additional non trivial technical developments.

\subsubsection{Construction of a solution for the operator with shaken co- efficients}

We start with the construction of the operator with shaken coefficients. Given $\epsilon>0$ and a (uniformly) strictly positive continuous map $\kappa$ with linear growth, that will be defined later on, let us introduce a family of perturbations of the operator appearing in (1.17):

$$
F_{\kappa}^{\epsilon}(t, x, q, M):=\min _{x^{\prime} \in D_{\kappa}^{\epsilon}(x)} \min \left\{-q-\frac{\sigma^{2}\left(x^{\prime}\right) M}{2\left(1-f\left(x^{\prime}\right) M\right)}, \bar{\gamma}\left(x^{\prime}\right)-M\right\},
$$


where

$$
D_{\kappa}^{\epsilon}(x):=\left\{x^{\prime} \in \mathbb{R}:\left(x-x^{\prime}\right) / \kappa\left(x^{\prime}\right) \in[-\epsilon, \epsilon]\right\} .
$$

For ease of notation, we set

$$
F_{\kappa}^{\epsilon}[\varphi](t, x):=F_{\kappa}^{\epsilon}\left(t, x, \partial_{t} \varphi(t, x), \partial_{x x}^{2} \varphi(t, x)\right),
$$

whenever $\varphi$ is smooth.

Remark 2.1. For later use, note that the map $M \in(-\infty, \bar{\gamma}(x)] \mapsto \frac{\sigma^{2}(x) M}{2(1-f(x) M)}$ is non-decreasing and convex, for each $x \in \mathbb{R}$, recall 1.13). Hence, $(q, M) \in$ $\mathbb{R} \times(-\infty, \bar{\gamma}(x)] \mapsto F_{\kappa}^{\epsilon}(\cdot, q, M)$ is concave and non-increasing in $M$, for all $\epsilon \geq 0$. This is fundamental for our smoothing approach to go through.

We also modify the original terminal condition $\hat{g}$ by using an approximating sequence whose elements are affine for large values of $|x|$.

Lemma 2.2. For all $K>0$ there exists a uniformly continuous map $\hat{g}_{K}$ and $x_{K} \geq K$ such that

- $\hat{g}_{K}$ is affine on $\left[x_{K}, \infty\right)$ and on $\left(-\infty,-x_{K}\right]$

- $\hat{g}_{K}=\hat{g}$ on $[-K, K]$

- $\hat{g}_{K} \geq \hat{g}$

- $\hat{g}_{K}-\bar{\Gamma}$ is concave for any $C^{2}$ function $\bar{\Gamma}$ satisfying $\partial_{x x}^{2} \bar{\Gamma}=\bar{\gamma}$.

Moreover, $\left(\hat{g}_{K}\right)_{K>0}$ is uniformly bounded by a map with linear growth and converges to $\hat{g}$ uniformly on compact sets.

Proof. Fix a $C^{2}$ function $\bar{\Gamma}^{\circ}$ satisfying $\partial_{x x}^{2} \bar{\Gamma}^{\circ}=\bar{\gamma}$. By definition, $\hat{g}-\bar{\Gamma}^{\circ}$ is concave. Let us consider an element $\Delta^{+}$(resp. $\Delta^{-}$) of its super-differential at $K$ (resp. $-K$ ). Set

$$
\begin{aligned}
\hat{g}_{K}^{\circ}(x):= & \hat{g}(x) \mathbf{1}_{[-K, K]}(x) \\
& +\left[\hat{g}(K)+\left(\Delta^{+}+\partial_{x} \bar{\Gamma}^{\circ}(K)\right)(x-K)\right] \mathbf{1}_{(K, \infty)}(x) \\
& +\left[\hat{g}(-K)+\left(\Delta^{-}+\partial_{x} \bar{\Gamma}^{\circ}(-K)\right)(x+K)\right] \mathbf{1}_{(-\infty,-K)}(x) .
\end{aligned}
$$

Consider now another $C^{2}$ function $\bar{\Gamma}$ satisfying $\partial_{x x}^{2} \bar{\Gamma}=\bar{\gamma}$. Since $\bar{\Gamma}^{\circ}$ and $\bar{\Gamma}$ differ only by an affine map, the concavity of $\hat{g}_{K}^{\circ}-\bar{\Gamma}$ is equivalent to that of $\hat{g}_{K}^{\circ}-\bar{\Gamma}^{\circ}$. The concavity of the latter follows from the definition of $\hat{g}_{K}^{\circ}$, as the superdiffential of $\hat{g}_{K}^{\circ}-\bar{\Gamma}^{\circ}$ is non-increasing by construction. In particular, $\hat{g}_{K}^{\circ}-\bar{\Gamma}^{\circ} \geq \hat{g}-\bar{\Gamma}^{\circ}$ and 
therefore $\hat{g}_{K}^{\circ} \geq \hat{g}$.

We finally define $\hat{g}_{K}$ by

$$
\hat{g}_{K}=\min \left\{\hat{g}_{K}^{\circ},\left(2 c_{0}+c_{1}|\cdot|-\bar{\Gamma}^{\circ}\right)^{\text {conc }}+\bar{\Gamma}^{\circ}\right\},
$$

with $c_{0}>0$ and $c_{1} \geq 0$ such that

$$
-c_{0} \leq \hat{g}(x) \leq c_{0}+c_{1}|x|, x \in \mathbb{R},
$$

recall Remark 1.6. The function $\hat{g}_{K}$ has the same linear growth as $2 c_{0}+c_{1}|\cdot|$, by the same reasoning as in Remark 1.6. Since $2 c_{0}>c_{0}, \hat{g}_{K}=\hat{g}_{K}^{\circ}=\hat{g}$ on $[-K, K]$. Furthermore, as the minimum of two concave functions is concave, so is $\hat{g}_{K}-\bar{\Gamma}$ for any $C^{2}$ function $\bar{\Gamma}$ satisfying $\partial_{x x}^{2} \bar{\Gamma}=\bar{\gamma}$. The other assertions are immediate.

We now set

$$
\hat{g}_{K}^{\epsilon}:=\hat{g}_{K}+\epsilon
$$

and consider the equation

$$
F_{\kappa}^{\epsilon}[\varphi] \mathbf{1}_{[0, T)}+\left(\varphi-\hat{g}_{K}^{\epsilon}\right) \mathbf{1}_{\{T\}}=0 .
$$

We then choose $\kappa$ and $\epsilon_{\circ} \in(0,1)$ such that

$$
\begin{gathered}
\kappa \in C^{\infty} \text { with bounded derivatives of all orders, } \\
\begin{aligned}
\inf \kappa>0 \text { and } \kappa & =\left|\hat{g}_{K}\right|+1 \text { on }\left(-\infty,-x_{K}\right] \cup\left[x_{K}, \infty\right), \\
& -1 / \epsilon_{\circ}<\partial_{x} \kappa<1 / \epsilon_{\circ},
\end{aligned}
\end{gathered}
$$

in which $x_{K} \geq K$ is defined in Lemma 2.2. We omit the dependence of $\kappa$ on $K$ for ease of notations.

Remark 2.3. For later use, note that the condition $\left|\partial_{x} \kappa\right|<1 / \epsilon_{\circ}$ ensures that the map $x \mapsto x+\epsilon \kappa(x)$ and $x \mapsto x-\epsilon \kappa(x)$ are uniformly strictly increasing for all $0 \leq \epsilon \leq \epsilon_{\circ}$. Also observe that $x_{n} \rightarrow x$ and $x_{n}^{\prime} \in D_{\kappa}^{\epsilon}\left(x_{n}\right)$, for all $n$, imply that $x_{n}^{\prime}$ converges to an element $x^{\prime} \in D_{\kappa}^{\epsilon}(x)$, after possibly passing to a subsequence. In particular, $F_{\kappa}^{\epsilon}$ is continuous.

When $\kappa \equiv 1$ and $\hat{g}_{K}^{\epsilon} \equiv \hat{g}+\epsilon,(2.4)$ corresponds to the operator in (1.17) with shaken coefficients, in the traditional terminology of [15]. The function $\kappa$ will be used below to handle the potential linear growth at infinity of $\hat{g}$. The introduction of the additional approximation $\hat{g}_{K}^{\epsilon}$ is motivated by the fact that the proof of Proposition 2.7 below requires an affine behavior at infinity. As already mentioned, these additional complications do not appear in [5] because their terminal condition is bounded. 
We now prove that 2.4 admits a viscosity solution that remains above the terminal condition $\hat{g}$ on a small time interval $\left[T-c_{\epsilon}^{K}, T\right]$. As already mentioned, we will later smooth this solution out with a regular kernel, so as to provide a smooth supersolution of (1.17).

Proposition 2.4. For all $\epsilon \in\left[0, \epsilon_{\circ}\right]$ and $K>0$, there exists a unique continuous viscosity solution $\overline{\mathrm{v}}_{\bar{\gamma}}^{\epsilon, K}$ of 2.4 that has linear growth. It satisfies

$$
\overline{\mathrm{v}}_{\bar{\gamma}}^{\epsilon, K} \geq \hat{g}_{K}+\epsilon / 2, \quad \text { on }\left[T-c_{\epsilon}^{K}, T\right] \times \mathbb{R},
$$

for some $c_{\epsilon}^{K} \in(0, T)$.

Moreover, $\left\{\left[\overline{\mathrm{v}}_{\bar{\gamma}}^{\epsilon}\right]^{+}, \epsilon \in\left[0, \epsilon_{\circ}\right], K>0\right\}$ is bounded by a map with linear growth, and $\left\{\left[\overline{\mathrm{v}}_{\bar{\gamma}}^{\epsilon, K}\right]^{-}, \epsilon \in\left[0, \epsilon_{\circ}\right], K>0\right\}$ is bounded by $\sup g^{-}$.

Proof. The proof is mainly a modification of the usual Perron's method, see [10, Section 4].

a. We first prove that there exists two continuous functions $\bar{w}$ and $\underline{w}$ with linear growth that are respectively super- and subsolution of 2.4 for any $\epsilon \in\left[0, \epsilon_{\circ}\right]$.

Since $\hat{g}_{K}^{\epsilon}=\hat{g}_{K}+\epsilon \geq g$ by Lemma 2.2 , it suffices to set

$$
\underline{w}:=\inf g>-\infty \text {, }
$$

see (1.16). To construct a supersolution $\bar{w}$, let us fix $\eta \in\left(0, \iota \wedge \inf f^{-1}\right)$ with $\iota$ as in 1.13 , set $\tilde{\Gamma}(x)=\eta x^{2} / 2$ and define $\tilde{g}=\left(\hat{g}_{K}^{\epsilon_{\circ}}-\tilde{\Gamma}\right)^{\text {conc }}+\tilde{\Gamma}$. Then, $\tilde{g} \geq \hat{g}_{K}^{\epsilon_{\circ}}$, while the same reasoning as in Remark 1.6 implies that $\tilde{g}$ shares the same linear growth as $\hat{g}_{K}^{\epsilon_{\circ}}$, see 2.3 and Lemma 2.2 . We then define $\bar{w}$ by

$$
\bar{w}(t, x)=\tilde{g}(x)+1+(T-t) A
$$

in which

$$
A:=\sup \frac{\sigma^{2} \bar{\gamma}}{2(1-f \bar{\gamma})} .
$$

The constant $A$ is finite, and $\bar{w}$ has the same linear growth as $\tilde{g}$, see (1.1)-(1.13). Since a concave function is a viscosity supersolution of $-\partial_{x x}^{2} \varphi \geq 0$, we deduce that $\tilde{g}$ is a viscosity supersolution of $\eta-\partial_{x x}^{2} \varphi \geq 0$. Then, $\bar{w}$ is a viscosity supersolution of

$$
\min \left\{-\partial_{t} \varphi-A, \eta-\partial_{x x}^{2} \varphi\right\} \geq 0 .
$$

Since $\bar{\gamma} \geq \iota \geq \eta$, it remains to use Remark 2.1 to conclude that $\bar{w}$ is a supersolution of (2.4). 
b. We now express $(2.4)$ as a single equation over the whole domain $[0, T] \times \mathbb{R}$ using the following definitions

$$
\begin{aligned}
& F_{\kappa,+}^{\epsilon, K}(t, x, r, q, M):=F_{\kappa}^{\epsilon}(t, x, q, M) \mathbf{1}_{[0, T)}+\max \left\{F_{\kappa}^{\epsilon}(t, x, q, M), r-\hat{g}_{K}^{\epsilon}(x)\right\} \mathbf{1}_{\{T\}} \\
& F_{\kappa,-}^{\epsilon, K}(t, x, r, q, M):=F_{\kappa}^{\epsilon}(t, x, q, M) \mathbf{1}_{[0, T)}+\min \left\{F_{\kappa}^{\epsilon}(t, x, q, M), r-\hat{g}_{K}^{\epsilon}(x)\right\} \mathbf{1}_{\{T\}} .
\end{aligned}
$$

As usual $F_{\kappa, \pm}^{\epsilon, K}[\varphi](t, x):=F_{\kappa, \pm}^{\epsilon, K}\left(t, x, \varphi(t, x), \partial_{t} \varphi(t, x), \partial_{x x}^{2} \varphi(t, x)\right)$. Recall that the formulations in terms of $F_{\kappa, \pm}^{\epsilon, K}$ lead to the same viscosity solutions as 2.4 (see Lemma 5.1 in the Appendix). This is the formulation to which we apply Perron's method. In view of a., the functions $\underline{w}$ and $\bar{w}$ are sub- and supersolution of $F_{\kappa,-}^{\epsilon, K}=0$ and $F_{\kappa,+}^{\epsilon, K}=0$. Define:

$$
\overline{\mathrm{V}}_{\bar{\gamma}}^{\epsilon, K}:=\sup \left\{v \in \mathrm{USC}: \underline{w} \leq v \leq \bar{w} \text { and } v \text { is a subsolution of } F_{\kappa,-}^{\epsilon, K}=0\right\},
$$

in which USC denotes the class of upper-semicontinuous maps. Then, the upper(resp. lower-)semicontinuous envelope $\left(\overline{\mathrm{v}}_{\bar{\gamma}}^{\epsilon, K}\right)^{*}\left(\operatorname{resp} .\left(\overline{\mathrm{v}}_{\bar{\gamma}}^{\epsilon, K}\right)_{*}\right)$ of $\overline{\mathrm{v}}_{\bar{\gamma}}^{\epsilon, K}$ is a viscosity subsolution of $F_{\kappa,-}^{\epsilon, K}[\varphi]=0$ (resp. supersolution of $F_{\kappa,+}^{\epsilon, K}[\varphi]=0$ ) with linear growth, recall the continuity property of Remark 2.3 and see e.g. [10, Section 4]. The comparison result of Theorem 2.11 stated below implies that

$$
\left(\overline{\mathrm{v}}_{\bar{\gamma}}^{\epsilon, K}\right)^{*}=\left(\overline{\mathrm{v}}_{\bar{\gamma}}^{\epsilon, K}\right)_{*}, \quad \text { on }[0, T] \times \mathbb{R} .
$$

Hence, $\overline{\mathrm{v}} \bar{\gamma}{ }_{\bar{\gamma}}$ is a continuous viscosity solution of 2.4 , recall Lemma 5.1. By construction, it has linear growth. Uniqueness in this class follows from Theorem 2.11 again.

c. It remains to prove 2.6. For this, we need a control on the behavior of $\overline{\mathrm{v}}_{\bar{\gamma}}^{\epsilon, K}$ as $t \rightarrow T$. It is enough to obtain it for a lower bound $v_{\epsilon, K}$ that we first construct. Let $\varphi$ be a test function such that

$$
\text { (strict) } \min _{[0, T) \times \mathbb{R}}\left(\overline{\mathrm{v}}_{\bar{\gamma}}^{\epsilon, K}-\varphi\right)=\left(\overline{\mathrm{v}}_{\bar{\gamma}}^{\epsilon, K}-\varphi\right)\left(t_{0}, x_{0}\right)
$$

for some $\left(t_{0}, x_{0}\right) \in[0, T) \times \mathbb{R}$. By the supersolution property,

$$
\min _{x^{\prime} \in D_{\kappa}^{\epsilon}\left(x_{0}\right)}\left\{\bar{\gamma}\left(x^{\prime}\right)-\partial_{x x}^{2} \varphi\left(t_{0}, x_{0}\right)\right\} \geq 0 .
$$

Recalling 1.1 and (1.13), this implies that, for $x^{\prime} \in D_{\kappa}^{\epsilon}\left(x_{0}\right)$,

$$
1-f\left(x^{\prime}\right) \partial_{x x}^{2} \varphi\left(t_{0}, x_{0}\right) \geq \iota f\left(x^{\prime}\right) \geq \iota \inf f=: \tilde{\iota}>0 .
$$


Using the supersolution property and the above inequalities yields

$$
\begin{aligned}
0 & \leq \min _{x^{\prime} \in D_{\kappa}^{\epsilon}\left(x_{0}\right)}\left\{-\partial_{t} \varphi\left(t_{0}, x_{0}\right)-\frac{\sigma^{2}\left(x^{\prime}\right) \partial_{x x}^{2} \varphi\left(t_{0}, x_{0}\right)}{2\left(1-f\left(x^{\prime}\right) \partial_{x x}^{2} \varphi\left(t_{0}, x_{0}\right)\right)}\right\} \\
& \leq \min _{x^{\prime} \in D_{\kappa}^{\epsilon}\left(x_{0}\right)}\left\{-\partial_{t} \varphi\left(t_{0}, x_{0}\right)-\frac{\sigma^{2}\left(x^{\prime}\right)\left[\partial_{x x}^{2} \varphi\left(t_{0}, x_{0}\right)-\bar{\gamma}\left(x_{0}\right)\right]}{2\left(1-f\left(x^{\prime}\right) \partial_{x x}^{2} \varphi\left(t_{0}, x_{0}\right)\right)}\right\} \\
& \leq-\partial_{t} \varphi\left(t_{0}, x_{0}\right)-\frac{\tilde{\sigma}^{2} \partial_{x x}^{2} \varphi\left(t_{0}, x_{0}\right)}{2 \tilde{\iota}}+\frac{\tilde{\sigma}^{2} \bar{\gamma}\left(x_{0}\right)}{2 \tilde{\iota}}
\end{aligned}
$$

where $\tilde{\sigma}:=\sup \sigma$.

Denote by $v_{\epsilon, K}$ the unique viscosity solution of

$$
\left\{-\partial_{t} \varphi-\frac{\tilde{\sigma}^{2} \partial_{x x}^{2} \varphi}{2 \tilde{\iota}}+\frac{\tilde{\sigma}^{2} \bar{\gamma}}{2 \tilde{\iota}}\right\} \mathbf{1}_{[0, T)}+\left(\varphi-\hat{g}_{K}^{\epsilon}\right) \mathbf{1}_{\{T\}}=0 .
$$

The comparison principle for (2.7) and the Feynman-Kac formula imply that

$$
\overline{\mathrm{V}}_{\bar{\gamma}}^{\epsilon, K}(t, x) \geq v_{\epsilon, K}(t, x)=\mathbb{E}\left[-\int_{0}^{T-t} \frac{\tilde{\sigma}^{2} \bar{\gamma}\left(S_{r}^{x}\right)}{2 \tilde{\iota}} d r+\hat{g}_{K}^{\epsilon}\left(S_{T-t}^{x}\right)\right]
$$

where

$$
S^{x}=x+\frac{\tilde{\sigma}}{\sqrt{\tilde{\iota}}} W .
$$

It remains to show that 2.6 holds for $v_{\epsilon, K}$ in place of $\overline{\mathrm{v}}_{\bar{\gamma}, K}$. The argument is standard. Since $\hat{g}_{K}$ is uniformly continuous, see Lemma 2.2 we can find $B_{\varepsilon}^{K}>0$ such that

$$
\left|\hat{g}_{K}^{\epsilon}\left(S_{T-t}^{x}\right)-\hat{g}_{K}^{\epsilon}(x)\right| \mathbf{1}_{\left\{\left|S_{T-t}^{x}-x\right| \leq B_{\varepsilon}^{K}\right\}} \leq \varepsilon
$$

for all $\varepsilon>0$. We now consider the case $\left|S_{T-t}^{x}-x\right|>B_{\varepsilon}^{K}$. Let $C>0$ denote a generic constant that does not depend on $(t, x)$ but can change from line to line. Because, $\hat{g}_{K}$ is affine on $\left[x_{K}, \infty\right)$ and on $\left(-\infty,-x_{K}\right]$, see Lemma 2.2.

$$
\mathbb{E}\left[\left|\hat{g}_{K}^{\epsilon}\left(S_{T-t}^{x}\right)-\hat{g}_{K}^{\epsilon}(x)\right| \mathbf{1}_{\left\{S_{T-t}^{x} \geq x_{K}\right\}}\right] \leq C(T-t)^{\frac{1}{2}} \text { if } x \geq x_{K}
$$

and

$$
\mathbb{E}\left[\left|\hat{g}_{K}^{\epsilon}\left(S_{T-t}^{x}\right)-\hat{g}_{K}^{\epsilon}(x)\right| \mathbf{1}_{\left\{S_{T-t}^{x} \leq-x_{K}\right\}}\right] \leq C(T-t)^{\frac{1}{2}} \text { if } x \leq-x_{K} .
$$

On the other hand, by linear growth of $\hat{g}_{K}^{\epsilon}$, if $x<x_{K}$, then

$$
\begin{aligned}
& \mathbb{E}\left[\left|\hat{g}_{K}^{\epsilon}\left(S_{T-t}^{x}\right)-\hat{g}_{K}^{\epsilon}(x)\right| \mathbf{1}_{\left\{S_{T-t}^{x} \geq x_{K}\right\}} \mathbf{1}_{\left\{\left|S_{T-t}^{x}-x\right| \geq B_{\varepsilon}^{K}\right\}}\right] \\
& \leq \mathbb{E}\left[\left|\hat{g}_{K}^{\epsilon}\left(S_{T-t}^{x}\right)-\hat{g}_{K}^{\epsilon}(x)\right|^{2}\right]^{\frac{1}{2}} \mathbb{P}\left[\left|S_{T-t}^{x}-x\right| \geq\left|x_{K}-x\right| \vee B_{\varepsilon}^{K}\right]^{\frac{1}{2}} \\
& \leq C \frac{(1+|x|)(T-t)^{\frac{1}{2}}}{\left|x_{K}-x\right| \vee B_{\varepsilon}^{K}} \leq \frac{C}{B_{\varepsilon}^{K}}(T-t)^{\frac{1}{2}} .
\end{aligned}
$$


The (four) remaining cases are treated similarly, and we obtain

$$
\mathbb{E}\left[\left|\hat{g}_{K}^{\epsilon}\left(S_{T-t}^{x}\right)-\hat{g}_{K}^{\epsilon}(x)\right|\right] \leq \frac{C}{B_{\varepsilon}^{K}}(T-t)^{\frac{1}{2}}+\varepsilon .
$$

Since $\bar{\gamma}$ is bounded, this shows that

$$
\left|v_{\epsilon, K}(t, x)-\hat{g}_{K}^{\epsilon}(x)\right| \leq \frac{C}{B_{\varepsilon}^{K}}(T-t)^{\frac{1}{2}}+\varepsilon
$$

for $t \in[T-1, T]$. Hence the required result for $v_{\epsilon, K}$. Since $\overline{\mathrm{v}}_{\bar{\gamma}}^{\epsilon, K} \geq v_{\epsilon, K}$, this concludes the proof of 2.6 .

For later use, note that, by stability, $\overline{\mathrm{v}}_{\bar{\gamma}}^{\epsilon, K}$ converges to a solution of 1.17 when $\epsilon \rightarrow 0$ and $K \rightarrow \infty$.

Proposition 2.5. As $\epsilon \rightarrow 0$ and $K \rightarrow \infty, \overline{\mathrm{v}}_{\bar{\gamma}}^{\epsilon, K}$ converges to a function $\overline{\mathrm{v}}_{\bar{\gamma}}$ that is the unique viscosity solution of (1.17) with linear growth.

Proof. The family of functions $\left\{\overline{\mathrm{v}}_{\bar{\gamma}}^{\epsilon, K}, \epsilon \in\left(0, \epsilon_{\circ}\right], K>0\right\}$ is uniformly bounded by a map with linear growth, see Proposition 2.4. In view of the comparison result of Theorem 2.11 below, it suffices to apply [2, Theorem 4.1].

Remark 2.6. The bounds on $\overline{\mathrm{v}}_{\bar{\gamma}}$ can be made explicit, which can be useful to design a numerical scheme, see Section 4.1 below. First, as a by-product of the proof of Proposition 2.4. $\overline{\mathrm{v}}_{\bar{\gamma}}^{\epsilon, K} \geq \inf g$. Passing to the limit as $\epsilon \rightarrow 0$ and $K \rightarrow \infty$ leads to

$$
\overline{\mathrm{v}}_{\bar{\gamma}} \geq \inf g=: \underline{w} .
$$

We have also obtained that

$$
\overline{\mathrm{V}}_{\bar{\gamma}}^{\epsilon, K} \leq\left(\hat{g}_{K}^{\epsilon_{\circ}}-\tilde{\Gamma}\right)^{\mathrm{conc}}+\tilde{\Gamma}+1+A
$$

in which $x \mapsto \tilde{\Gamma}(x)=\eta x^{2} / 2$ for some $\eta \in\left(0, \iota \wedge \inf f^{-1}\right)$ with $\iota$ as in 1.13), and $A:=T \sup \left(\sigma^{2} \bar{\gamma} /[2(1-f \bar{\gamma})]\right)$. On the other hand, 2.2 implies

$$
\hat{g}_{K}^{\epsilon_{\circ}} \leq 1+\left(2 c_{0}+c_{1}|\cdot|-\bar{\Gamma}^{\circ}\right)^{\text {conc }}+\bar{\Gamma}^{\circ}
$$

for $\bar{\Gamma}^{\circ}$ such that $\partial_{x x}^{2} \bar{\Gamma}^{\circ}=\bar{\gamma}$. Then,

$$
\begin{aligned}
\overline{\mathrm{v}}_{\bar{\gamma}}^{\epsilon, K} & \leq\left(1+\left(2 c_{0}+c_{1}|\cdot|-\bar{\Gamma}^{\circ}\right)^{\mathrm{conc}}+\bar{\Gamma}^{\circ}-\tilde{\Gamma}\right)^{\mathrm{conc}}+\tilde{\Gamma}+1+A \\
& \leq\left(1+\left(2 c_{0}+c_{1}|\cdot|-\tilde{\Gamma}\right)^{\mathrm{conc}}+\tilde{\Gamma}-\tilde{\Gamma}\right)^{\mathrm{conc}}+\tilde{\Gamma}+1+A \\
& =\left(1+2 c_{0}+c_{1}|\cdot|-\tilde{\Gamma}\right)^{\mathrm{conc}}+\tilde{\Gamma}+1+A=: \bar{w}
\end{aligned}
$$


and

$$
\overline{\mathrm{v}}_{\bar{\gamma}} \leq \bar{w}
$$

The function $\bar{w}$ defined above can be computed explicitly by arguing as in Remark 1.6.

Also note that (2.2) and the arguments of Remark 1.6 imply that there exists a constant $C>0$ such that

$$
\limsup _{|x| \rightarrow \infty}\left|\overline{\mathrm{v}}_{\bar{\gamma}}^{\epsilon, K}(x)\right| /\left(1+\left|\hat{g}_{K}(x)\right|\right) \leq C, \text { for all } \epsilon \in\left[0, \epsilon_{\circ}\right] \text { and } K>0 .
$$

\subsubsection{Regularization and verification}

Prior to applying our verification argument, it remains to smooth out the function

$\overline{\mathrm{v}}_{\bar{\gamma}}^{\epsilon, K}$. This is similar to [5, Section 3], but here again the fact that $\hat{g}$ may not be bounded incurs additional difficulties. In particular, we need to use a kernel with a space dependent window.

We first fix a smooth kernel

$$
\psi_{\delta}:=\delta^{-2} \psi(\cdot / \delta)
$$

in which $\delta>0$ and $\psi \in C_{b}^{\infty}$ is a non-negative function with the closure of its support $[-1,0] \times[-1,1]$ that integrates to 1 , and such that

$$
\int y \psi(\cdot, y) d y=0
$$

Let us set

$$
\overline{\mathrm{v}}_{\bar{\gamma}}^{\epsilon, K, \delta}(t, x):=\int_{\mathbb{R} \times \mathbb{R}} \overline{\mathrm{v}}_{\bar{\gamma}}^{\epsilon, K}\left(\left[t^{\prime}\right]^{+}, x^{\prime}\right) \frac{1}{\kappa(x)} \psi_{\delta}\left(t^{\prime}-t, \frac{x^{\prime}-x}{\kappa(x)}\right) d t^{\prime} d x^{\prime} .
$$

We recall that $\kappa$ enters into the definition of $F_{\kappa}^{\epsilon}$ and satisfies (2.5).

The following shows that $\overline{\mathrm{v}}_{\bar{\gamma}}^{\epsilon, K, \delta}$ is a smooth supersolution of 1.17 with a space gradient admitting bounded derivatives. This is due to the space dependent rescaling of the window by $\kappa$ and will be crucial for our verification arguments.

Proposition 2.7. For all $0<\epsilon<\epsilon_{\circ}$ and $K>0$ large enough, there exists $\delta_{\circ}>0$ such that $\overline{\mathrm{v}}_{\bar{\gamma}}^{\epsilon, K, \delta}$ is a $C^{\infty}$ supersolution of 1.17 for all $0<\delta<\delta_{\circ}$. It has linear growth and $\partial_{x} \overline{\mathrm{v}} \bar{\gamma}, K, \delta$ has bounded derivatives of any order.

Proof. a. It follows from $(2.5)$ and $(2.8)$ that

$$
\limsup _{|x| \rightarrow \infty}\left|\overline{\mathrm{v}}_{\bar{\gamma}}^{\epsilon, K}(x)\right| /(1+|\kappa(x)|)<\infty .
$$


Direct computations and 2.5 then show that $\overline{\mathrm{v}} \bar{\gamma}, K, \delta$ has linear growth and that all derivatives of $\partial_{x} \overline{\mathrm{v}}_{\bar{\gamma}}^{\epsilon, K, \delta}$ are uniformly bounded.

b. We now prove the supersolution property inside the parabolic domain. Since the proof is very close to that of [5, Theorem 3.3], we only provide the arguments that require to be adapted, and refer to their proof for other elementary details. Fix $\ell>0$ and set

$$
v_{\ell}(t, x):=\overline{\mathrm{v}}_{\bar{\gamma}}^{\epsilon, K, \delta}(t,(-\ell) \vee x \wedge \ell) .
$$

We omit the superscripts that are superfluous in this proof. Given $k \geq 1$, set

$$
v_{\ell, k}(z):=\inf _{z^{\prime} \in[0, T] \times \mathbb{R}}\left(v_{\ell}\left(z^{\prime}\right)+k\left|z-z^{\prime}\right|^{2}\right) .
$$

Since $v_{\ell}$ is bounded and continuous, the infimum in the above is achieved by a point $\hat{z}_{\ell, k}(z)=\left(\hat{t}_{\ell, k}(z), \hat{x}_{\ell, k}(z)\right)$, and $v_{\ell, k}$ is bounded, uniformly in $k \geq 1$. This implies that we can find $C_{\ell}>0$, independent of $k$, such that

$$
\left|z-\hat{z}_{\ell, k}(z)\right|^{2} \leq C_{\ell} / k=:\left(\rho_{\ell, k}\right)^{2} .
$$

Moreover, a simple change of variables argument shows that, if $\varphi$ is a smooth function such that $v_{\ell, k}-\varphi$ achieves a minimum at $z \in[0, T) \times(-\ell, \ell)$, then

$$
\left(\partial_{t} \varphi, \partial_{x} \varphi, \partial_{x x}^{2} \varphi\right)(z) \in \overline{\mathcal{P}}^{-} v_{\ell}\left(\hat{z}_{\ell, k}(z)\right)
$$

where $\overline{\mathcal{P}}^{-} v_{\ell}\left(\hat{z}_{\ell, k}(z)\right)$ denotes the closed parabolic subjet of $v_{\ell}$ at $\hat{z}_{\ell, k}(z)$; see e.g. [10] for the definition. Then, Proposition 2.4 implies that $v_{\ell, k}$ is a supersolution of

$$
\min _{x^{\prime} \in D_{\kappa}^{\epsilon}\left(\hat{x}_{\ell, k}\right)} \min \left\{-\partial_{t} \varphi-\frac{\sigma^{2}\left(x^{\prime}\right) \partial_{x x}^{2} \varphi}{2\left(1-f\left(x^{\prime}\right) \partial_{x x}^{2} \varphi\right)}, \bar{\gamma}\left(x^{\prime}\right)-\partial_{x x}^{2} \varphi\right\} \geq 0
$$

on $\left[\rho_{\ell, k}, T-\rho_{\ell, k}\right) \times\left(-\ell+\rho_{\ell, k}, \ell-\rho_{\ell, k}\right)$. We next deduce from 2.11$)$ that $x^{\prime} \in D_{\kappa}^{\epsilon / 2}(x)$ implies

$$
-\frac{\epsilon}{2} \kappa\left(x^{\prime}\right)-C_{\ell} / k^{\frac{1}{2}} \leq \hat{x}_{\ell, k}(t, x)-x^{\prime} \leq \frac{\epsilon}{2} \kappa\left(x^{\prime}\right)+C_{\ell} / k^{\frac{1}{2}} .
$$

Since inf $\kappa>0$, this shows that $x^{\prime} \in D_{\kappa}^{\epsilon}\left(\hat{x}_{\ell, k}(t, x)\right)$ for $k$ large enough with respect to $\ell$. Hence, $v_{\ell, k}$ is a supersolution of

$$
\min _{x^{\prime} \in D_{\kappa}^{\epsilon / 2}} \min \left\{-\partial_{t} \varphi-\frac{\sigma^{2}\left(x^{\prime}\right) \partial_{x x}^{2} \varphi}{2\left(1-f\left(x^{\prime}\right) \partial_{x x}^{2} \varphi\right)}, \bar{\gamma}\left(x^{\prime}\right)-\partial_{x x}^{2} \varphi\right\} \geq 0
$$

on $\left[\rho_{\ell, k}, T-\rho_{\ell, k}\right) \times\left(-\ell+\rho_{\ell, k}, \ell-\rho_{\ell, k}\right)$. 
We now argue as in [13]. Since $v_{\ell, k}$ is semi-concave, there exist $\partial_{x x}^{2, a b s} v_{\ell, k} \in L^{1}$ and a Lebesgue-singular negative Radon measure $\partial_{x x}^{2, s i n g} v_{\ell, k}$ such that

$$
\partial_{x x}^{2} v_{\ell, k}(d z)=\partial_{x x}^{2, a b s} v_{\ell, k}(z) d z+\partial_{x x}^{2, s i n g} v_{\ell, k}(d z) \text { in the distribution sense }
$$

and

$$
\left(\partial_{t} v_{\ell, k}, \partial_{x} v_{\ell, k}, \partial_{x x}^{2, a b s} v_{\ell, k}\right) \in \overline{\mathcal{P}}^{-} v_{\ell, k} \text { a.e. on }\left[\rho_{k}, T-\rho_{k}\right] \times\left(-\ell+\rho_{\ell, k}, \ell-\rho_{\ell, k}\right),
$$

see [14, Section 3]. Hence, the above implies that

$$
\min _{x^{\prime} \in D_{\kappa}^{\epsilon / 2}} \min \left\{-\partial_{t} v_{\ell, k}-\frac{\sigma^{2}\left(x^{\prime}\right) \partial_{x x}^{2, a b s} v_{\ell, k}}{2\left(1-f\left(x^{\prime}\right) \partial_{x x}^{2, a b s} v_{\ell, k}\right)}, \bar{\gamma}\left(x^{\prime}\right)-\partial_{x x}^{2, a b s} v_{\ell, k}\right\} \geq 0
$$

a.e. on $\left[\rho_{\ell, k}, T-\rho_{\ell, k}\right) \times\left(-\ell+\rho_{\ell, k}, \ell-\rho_{\ell, k}\right)$, or equivalently, by (2.1),

$$
\min \left\{-\partial_{t} v_{\ell, k}-\frac{\sigma^{2}(x) \partial_{x x}^{2, a b s} v_{\ell, k}}{2\left(1-f(x) \partial_{x x}^{2, a b s} v_{\ell, k}\right)}, \bar{\gamma}(x)-\partial_{x x}^{2, a b s} v_{\ell, k}\right\}\left(t^{\prime}, x^{\prime}\right) \geq 0
$$

for all $x$ and for a.e. $\left(t^{\prime}, x^{\prime}\right) \in\left[\rho_{\ell, k}, T-\rho_{\ell, k}\right) \times\left(-\ell+\rho_{\ell, k}, \ell-\rho_{\ell, k}\right)$ such that $2\left|x^{\prime}-x\right| \leq \epsilon \kappa(x)$. Take $0<\delta<\varepsilon / 2$. Integrating the previous inequality with respect to $\left(t^{\prime}, x^{\prime}\right)$ with the kernel function $\psi_{\delta}(\cdot, \cdot / \kappa) / \kappa$, using the concavity and monotonicity property of Remark 2.1 and the fact that $\partial_{x x}^{2, s i n g} v_{\ell, k}$ is non-positive, we obtain

$$
\min \left\{-\partial_{t} v_{\ell, k}^{\delta}-\frac{\sigma^{2} \partial_{x x}^{2} v_{\ell, k}^{\delta}}{2\left(1-f \partial_{x x}^{2} v_{\ell, k}^{\delta}\right)}, \bar{\gamma}-\partial_{x x}^{2} v_{\ell, k}^{\delta}\right\} \geq 0
$$

on $\left[\rho_{\ell, k}+\delta, T-\rho_{\ell, k}\right) \times\left(-x_{\ell, k}^{-}, x_{\ell, k}^{+}\right)$, in which

$$
v_{\ell, k}^{\delta}(t, x):=\int_{\mathbb{R} \times \mathbb{R}} v_{\ell, k}\left(\left[t^{\prime}\right]^{+}, x^{\prime}\right) \frac{1}{\kappa(x)} \psi_{\delta}\left(t^{\prime}-\cdot \frac{x^{\prime}-\cdot}{\kappa(x)}\right) d t^{\prime} d x^{\prime}
$$

and

$$
x_{\ell, k}^{+}+\frac{\delta}{2} \kappa\left(x_{\ell, k}^{+}\right)=\ell-\rho_{\ell, k} \text { and }-x_{\ell, k}^{-}-\frac{\delta}{2} \kappa\left(-x_{\ell, k}^{-}\right)=-\ell+\rho_{\ell, k} .
$$

The above are well defined, see Remark 2.3. By Remark 2.3 and $\sqrt{2.11}, \pm x_{\ell, k}^{ \pm} \rightarrow$ $\pm \infty$ and $\rho_{\ell, k} \rightarrow 0$ as $k \rightarrow \infty$ and then $\ell \rightarrow \infty$. Moreover, $v_{\ell, k}^{\delta} \rightarrow \overline{\mathrm{v}}_{\bar{\gamma}}^{\epsilon, K, \delta}$ as $k \rightarrow \infty$ and then $\ell \rightarrow \infty$, and the derivatives also converge. Hence, 2.12 implies that $\overline{\mathrm{v}}_{\bar{\gamma}}^{\epsilon, K, \delta}$ is a supersolution of 1.17 on $[\delta, T) \times \mathbb{R}$.

c. We conclude by discussing the boundary condition at $T$. We know from Proposition 2.4 that

$$
\overline{\mathrm{v}}_{\bar{\gamma}}^{\epsilon, K} \geq \hat{g}_{K}+\epsilon / 2, \quad \text { on }\left[T-c_{\epsilon}^{K}, T\right] \times \mathbb{R} \text {. }
$$


Since $\hat{g}$ is uniformly continuous, see 1.16 , so is $\hat{g}_{K}$, and therefore $\overline{\mathbf{v}} \bar{\gamma}, K, \delta(T, \cdot) \geq \hat{g}_{K}$ on the compact set $\left[-2 x_{K}, 2 x_{K}\right]$ for $\delta>0$ small enough with respect to $\epsilon$, see Lemma 2.2 for the definition of $x_{K} \geq K$. Now observe that $x \geq 2 x_{K}$ and $\left|x^{\prime}-x\right| \leq$ $\delta \kappa(x)$ imply that $x^{\prime} \geq 2 x_{K}\left(1-\delta c_{1}^{K}\right)-\delta c_{0}^{K}$ in which $c_{1}^{K}$ and $c_{0}^{K}$ are constants. This actually follows from the affine behavior of $\kappa$ on $\left[x_{K}, \infty\right)$, see (2.5) and Lemma 2.2. For $\delta$ small enough, we then obtain $x^{\prime} \geq x_{K}$. Since $\hat{g}_{K}$ is affine on $\left[x_{K}, \infty\right)$, and since $\psi$ is symmetric in its second argument, see $(2.9)$, it follows that

$$
\overline{\mathrm{v}}_{\bar{\gamma}}^{\epsilon, K, \delta}(T, x) \geq \int_{\mathbb{R} \times \mathbb{R}} \hat{g}_{K}\left(x^{\prime}\right) \frac{1}{\kappa(x)} \psi_{\delta}\left(t^{\prime}-T, \frac{x^{\prime}-x}{\kappa(x)}\right) d t^{\prime} d x^{\prime}=\hat{g}_{K}(x)
$$

for all $x \geq 2 x_{K}$. This also holds for $x \leq-2 x_{K}$, by the same arguments.

We can now use a verification argument and provide the main result of this section.

Theorem 2.8. Let $\overline{\mathrm{v}}_{\bar{\gamma}}$ be defined as in Proposition 2.5. It has linear growth. Moreover, $\overline{\mathrm{v}}_{\bar{\gamma}} \geq \mathrm{v}_{\bar{\gamma}}$ on $[0, T] \times \mathbb{R}$.

Proof. The linear growth property has already been stated in Proposition 2.5 We now show that $\overline{\mathrm{v}}_{\bar{\gamma}} \geq \mathrm{v} \bar{\gamma}$ by applying a verification argument to $\overline{\mathrm{v}} \bar{\gamma}, K, \delta$. From now on $0<\epsilon \leq \epsilon_{\circ}$ in which $\epsilon_{\circ}$ is as in (2.5). The parameters $K, \delta>0$ are chosen as in Proposition 2.7.

Fix $(t, x) \in(0, T) \times \mathbb{R}$ and $\delta \in(0, t \wedge \epsilon)$. Let $(X, Y, V)$ be defined as in (1.6)1.2 -1.7 with $\left(x, \partial_{x} \overline{\mathrm{v}}_{\bar{\gamma}}^{\epsilon, K, \delta}(t, x), \overline{\mathrm{v}}_{\bar{\gamma}}^{\epsilon, K, \delta}(t, x)-\partial_{x} \overline{\mathrm{v}}_{\bar{\gamma}}^{\epsilon, K, \delta}(t, x) x\right)$ as initial condition at $t$, and for the Markovian controls

$$
\begin{aligned}
& \hat{a}=\left(\frac{\sigma \partial_{x x}^{2} \overline{\mathrm{v}}_{\bar{\gamma}}^{\epsilon, K, \delta}}{1-f \partial_{x x}^{2} \overline{\mathrm{v}}_{\bar{\gamma}}^{\epsilon, K, \delta}}\right)(\cdot, X) \\
& \hat{b}=\left(\frac{\partial_{t x}^{2} \overline{\mathrm{v}}_{\bar{\gamma}}^{\epsilon, K, \delta}+\partial_{x x}^{2} \overline{\mathrm{v}}_{\bar{\gamma}}^{\epsilon, K, \delta}\left(\mu+\hat{a} \sigma f^{\prime}\right)+\frac{1}{2} \partial_{x x x}^{3} \overline{\mathrm{v}}_{\bar{\gamma}}^{\epsilon, K, \delta}(\sigma+\hat{a} f)^{2}}{1-f \partial_{x x}^{2} \overline{\mathrm{v}}_{\bar{\gamma}}^{\epsilon, K, \delta}}\right)(\cdot, X) .
\end{aligned}
$$

By definition of $F,(1.13)$ and (1.1), the above is well-defined as the denominators are always bigger than inf $f \iota>0$. All the involved functions being bounded and Lipschitz, see Proposition 2.7, it is easy to check that a solution to the corresponding stochastic differential equation exists, and that $(\hat{a}, \hat{b}) \in \mathcal{A}^{\circ}$. Direct computations then show that $Y=\partial_{x} \overline{\mathrm{v}}_{\bar{\gamma}}^{\epsilon, K, \delta}(\cdot, X)$. Moreover, the fact that $\overline{\mathrm{v}}_{\bar{\gamma}}^{\epsilon, K, \delta}$ is a supersolution of $F[\varphi]=0$ on $[t, T] \times \mathbb{R}$ ensures that the gamma constraint 1.12 holds, for some $k \geq 1$, and that

$$
-\partial_{t} \overline{\mathrm{v}}_{\bar{\gamma}}^{\epsilon, K, \delta}(\cdot, X)-\frac{1}{2} \sigma(X) \hat{a} \geq 0 \text { on }[t, T) .
$$


The last inequality combined with the definition of $\hat{a}$ implies

$$
\begin{aligned}
\frac{1}{2} f(X) \hat{a}^{2} & \geq \partial_{t} \overline{\mathrm{v}}_{\bar{\gamma}}^{\epsilon, K, \delta}(\cdot, X)+\frac{1}{2}(\sigma(X)+f(X) \hat{a}) \hat{a} \\
& =\partial_{t} \overline{\mathrm{v}}_{\bar{\gamma}}^{\epsilon, K, \delta}(\cdot, X)+\frac{1}{2}\left(\sigma_{X}^{\hat{a}}(X)\right)^{2} \partial_{x x}^{2} \overline{\mathrm{v}}_{\bar{\gamma}}^{\epsilon, K, \delta}(\cdot, X) \text { on }[t, T)
\end{aligned}
$$

Hence,

$$
\begin{aligned}
V_{T} & =\overline{\mathrm{v}}_{\bar{\gamma}}^{\epsilon, K, \delta}(t, x)+\frac{1}{2} \int_{t}^{T} f\left(X_{u}\right) \hat{a}_{u}^{2} d u+\int_{t}^{T} \partial_{x} \overline{\mathrm{v}}_{\bar{\gamma}}^{\epsilon, K, \delta}\left(u, X_{u}\right) d X_{u} \\
& \geq \overline{\mathrm{v}}_{\bar{\gamma}}^{\epsilon, K, \delta}(t, x)+\int_{t}^{T} d \overline{\mathrm{v}}_{\bar{\gamma}}^{\epsilon, K, \delta}\left(u, X_{u}\right) \\
& =\overline{\mathrm{v}}_{\bar{\gamma}}^{\epsilon, K, \delta}\left(T, X_{T}\right) \geq g\left(X_{T}\right),
\end{aligned}
$$

in which the last inequality follows from Proposition 2.7 again.

It remains to pass to the limit $\delta, \epsilon \rightarrow 0$. By Proposition 2.4. $\overline{\mathrm{v}}_{\bar{\gamma}}^{\epsilon, K}$ is continuous, so that $\overline{\mathrm{v}}_{\bar{\gamma}}^{\epsilon, K, \delta}$ converges pointwise to $\overline{\mathrm{v}}_{\bar{\gamma}}^{\epsilon, K}$ as $\delta \rightarrow 0$. By Proposition 2.5. $\overline{\mathrm{v}}_{\bar{\gamma}}^{\epsilon, K}$ converges pointwise to $\overline{\mathrm{v}}_{\bar{\gamma}}$ as $\epsilon \rightarrow 0$ and $K \rightarrow \infty$. In view of the above this implies the required result: $\overline{\mathrm{v}}_{\bar{\gamma}} \geq \mathrm{v} \bar{\gamma}$.

Remark 2.9. Note that, in the above proof, we have constructed a super-hedging strategy in $\mathcal{A}_{k, \bar{\gamma}}(t, x)$ and starting with $\left|Y_{t}\right| \leq k$, for some $k \geq 1$ which can be chosen in a uniform way with respect to $(t, x)$, while $\overline{\mathrm{v}}_{\bar{\gamma}}^{\epsilon, K, \delta}$ has linear growth.

\subsubsection{Comparison principle}

We provide here the comparison principle that was used several times in the above. Before stating it, let us make the following observation, based on direct computations. Recall (1.1) and (1.13).

Proposition 2.10. Fix $\rho>0$. Consider the map

$$
(t, x, M) \in[0, T] \times \mathbb{R} \times \mathbb{R} \mapsto \Psi(t, x, M)=\frac{\sigma^{2}(x) M}{2\left(e^{\rho t}-f(x) M\right)} .
$$

Then, $M \mapsto \Psi(t, x, M)$ is continuous, uniformly in $(t, x)$, on

$$
O:=\left\{(t, x, M) \in[0, T] \times \mathbb{R} \times \mathbb{R}: M \leq e^{\rho t} \bar{\gamma}(x)\right\} .
$$

Moreover, there exists $L>0$ such that $x \mapsto \Psi(t, x, M)$ is L-Lipschitz on $O$. 
Theorem 2.11. Fix $\epsilon \in\left[0, \epsilon_{\circ}\right]$. Let $U$ (resp. $V$ ) be a upper semicontinuous viscosity subsolution (resp. lower semicontinuous supersolution) of $F_{\kappa}^{\epsilon}=0$ on $[0, T) \times \mathbb{R}$. Assume that $U$ and $V$ have linear growth and that $U \leq V$ on $\{T\} \times \mathbb{R}$, then $U \leq V$ on $[0, T] \times \mathbb{R}$.

Proof. Set $\hat{U}(t, x):=e^{\rho t} U(t, x), \hat{V}(t, x):=e^{\rho t} V(t, x)$. Then, $\hat{U}$ and $\hat{V}$ are respectively sub- and supersolution of

$$
\min _{x^{\prime} \in D_{\kappa}^{\epsilon}} \min \left\{\rho \varphi-\partial_{t} \varphi-\frac{\sigma^{2}\left(x^{\prime}\right) \partial_{x x} \varphi}{2\left(e^{\rho t}-f\left(x^{\prime}\right) \partial_{x x} \varphi\right)}, e^{\rho t} \bar{\gamma}\left(x^{\prime}\right)-\partial_{x x} \varphi\right\}=0
$$

on $[0, T) \times \mathbb{R}$. For later use, note that the infimum over $D_{\kappa}^{\epsilon}$ is achieved in the above, by the continuity of the involved functions.

If $\sup _{[0, T] \times \mathbb{R}}(\hat{U}-\hat{V})>0$, then we can find $\lambda \in(0,1)$ such that $\sup _{[0, T] \times \mathbb{R}}(\hat{U}-$ $\left.\hat{V}_{\lambda}\right)>0$ with $\hat{V}_{\lambda}:=\lambda \hat{V}+(1-\lambda) w$, in which

$$
w(t, x):=(T-t) A+\left(c_{0}^{U}+c_{1}^{U}|\cdot|-\frac{\iota}{4}|\cdot|^{2}\right)^{\mathrm{conc}}(x)+\frac{\iota}{4}|x|^{2}
$$

with $c_{0}^{U}, c_{1}^{U}$ two constants such that $e^{\rho T}|U| \leq c_{0}^{U}+c_{1}^{U}|\cdot|$ and

$$
A:=\frac{1}{2} \sup \frac{\sigma^{2}}{1-\frac{\iota}{2} f} \frac{\iota}{2}
$$

where $\iota>0$ is as in 1.13 . Note that

$$
\hat{V}_{\lambda}(T, \cdot) \geq \hat{U}(T, \cdot),
$$

and that

$w$ is a viscosity supersolution of 2.13

$\hat{V}_{\lambda}$ is a viscosity supersolution of $\lambda \bar{\gamma}+(1-\lambda) \frac{\iota}{2}-\partial_{x x}^{2} \varphi \geq 0$.

Moreover, by Remark 2.1. $\hat{V}_{\lambda}$ is a supersolution of 2.13. Define for $\varepsilon>0$ and $n \geq 1$

$$
\Theta_{n}^{\varepsilon}:=\sup _{(t, x, y) \in[0, T] \times \mathbb{R}^{2}}\left[\hat{U}(t, x)-\hat{V}_{\lambda}(t, y)-\left(\frac{\varepsilon}{2}|x|^{2}+\frac{n}{2}|x-y|^{2}\right)\right]=: \eta>0,
$$

in which the last inequality holds for $n>0$ large enough and $\varepsilon>0$ small enough. Denote by $\left(t_{n}^{\varepsilon}, x_{n}^{\varepsilon}, y_{n}^{\varepsilon}\right)$ the point at which this supremum is achieved. By (2.14), it must hold that $t_{n}^{\varepsilon}<T$, and, by standard arguments, see e.g., [10, Proposition 3.7],

$$
\lim _{n \rightarrow \infty} n\left|x_{n}^{\varepsilon}-y_{n}^{\varepsilon}\right|^{2}=0
$$


Moreover, Ishii's lemma implies the existence of $\left(a_{n}^{\varepsilon}, M_{n}^{\varepsilon}, N_{n}^{\varepsilon}\right) \in \mathbb{R}^{3}$ such that

$$
\begin{gathered}
\left(a_{n}^{\varepsilon}, \varepsilon x^{\varepsilon}+n\left(x_{n}^{\varepsilon}-y_{n}^{\varepsilon}\right), M_{n}^{\varepsilon}\right) \in \overline{\mathcal{P}}^{2,+} \hat{U}\left(t_{n}^{\varepsilon}, x_{n}^{\varepsilon}\right) \\
\left(a_{n}^{\varepsilon},-n\left(x_{n}^{\varepsilon}-y_{n}^{\varepsilon}\right), N_{n}^{\varepsilon}\right) \in \overline{\mathcal{P}}^{2,-} \hat{V}_{\lambda}\left(t_{n}^{\varepsilon}, y_{n}^{\varepsilon}\right),
\end{gathered}
$$

in which $\overline{\mathcal{P}}^{2,+}$ and $\overline{\mathcal{P}}^{2,-}$ denote as usual the closed parabolic super- and subjets, see [10], and

$$
\left(\begin{array}{cc}
M_{n}^{\varepsilon} & 0 \\
0 & -N_{n}^{\varepsilon}
\end{array}\right) \leq R_{n}^{\varepsilon}+\frac{1}{n}\left(R_{n}^{\varepsilon}\right)^{2}=3 n\left(\begin{array}{cc}
1 & -1 \\
-1 & 1
\end{array}\right)+\left(\begin{array}{cc}
3 \varepsilon+\frac{\varepsilon^{2}}{n} & -\varepsilon \\
-\varepsilon & 0
\end{array}\right)
$$

with

$$
R_{n}^{\varepsilon}:=n\left(\begin{array}{cc}
1+\frac{\varepsilon}{n} & -1 \\
-1 & 1
\end{array}\right)
$$

In particular,

$$
M_{n}^{\varepsilon}-N_{n}^{\varepsilon} \leq \delta_{n}^{\varepsilon} \text { with } \delta_{n}^{\varepsilon}:=\varepsilon+\frac{\varepsilon^{2}}{n} .
$$

Then, by 2.15 and 1.13,

$$
0<(1-\lambda) \frac{\iota}{2} \leq e^{\rho t_{n}^{\varepsilon}} \bar{\gamma}\left(\hat{y}_{n}^{\varepsilon}\right)-N_{n}^{\varepsilon} \leq e^{\rho t_{n}^{\varepsilon}} \bar{\gamma}\left(\hat{y}_{n}^{\varepsilon}\right)-M_{n}^{\varepsilon}+\delta_{n}^{\varepsilon},
$$

in which $\hat{y}_{n}^{\varepsilon} \in D_{\kappa}^{\epsilon}\left(y_{n}^{\varepsilon}\right)$. In view of Remark 2.3 , this shows that $e^{\rho t_{n}^{\varepsilon}} \bar{\gamma}\left(\hat{x}_{n}^{\varepsilon}\right)-M_{n}^{\varepsilon}>0$ for some $\hat{x}_{n}^{\varepsilon} \in D_{\kappa}^{\epsilon}\left(x_{n}^{\varepsilon}\right)$, for $n$ large enough and $\varepsilon$ small enough, recall (2.17). Hence, the super- and subsolution properties of $\hat{V}_{\lambda}$ and $\hat{U}$ imply that we can find $u_{n}^{\varepsilon} \in$ $[-\epsilon, \epsilon]$ together with $\hat{y}_{n}^{\varepsilon}$ and $\hat{x}_{n}^{\varepsilon}$ such that

$$
\hat{y}_{n}^{\varepsilon}+u_{n}^{\varepsilon} \kappa\left(\hat{y}_{n}^{\varepsilon}\right)=y_{n}^{\varepsilon}, \hat{x}_{n}^{\varepsilon}+u_{n}^{\varepsilon} \kappa\left(\hat{x}_{n}^{\varepsilon}\right)=x_{n}^{\varepsilon}
$$

and

$$
\rho\left(\hat{U}\left(t_{n}^{\varepsilon}, x_{n}^{\varepsilon}\right)-\hat{V}_{\lambda}\left(t_{n}^{\varepsilon}, y_{n}^{\varepsilon}\right)\right) \leq \frac{\sigma^{2}\left(\hat{x}_{n}^{\varepsilon}\right) M_{n}^{\varepsilon}}{2\left(e^{\rho t_{n}^{\varepsilon}}-f\left(\hat{x}_{n}^{\varepsilon}\right) M_{n}^{\varepsilon}\right)}-\frac{\sigma^{2}\left(\hat{y}_{n}^{\varepsilon}\right) N_{n}^{\varepsilon}}{2\left(e^{\rho t_{n}^{\varepsilon}}-f\left(\hat{y}_{n}^{\varepsilon}\right) N_{n}^{\varepsilon}\right)} .
$$

By Remark 2.1 and 2.18), this shows that

$$
\begin{aligned}
& \rho\left(\hat{U}\left(t_{n}^{\varepsilon}, x_{n}^{\varepsilon}\right)-\hat{V}_{\lambda}\left(t_{n}^{\varepsilon}, y_{n}^{\varepsilon}\right)\right) \\
& \leq \frac{\sigma^{2}\left(\hat{x}_{n}^{\varepsilon}\right)\left(N_{n}^{\varepsilon}+\delta_{n}^{\varepsilon}\right)}{2\left(e^{\rho t_{n}^{\varepsilon}}-f\left(\hat{x}_{n}^{\varepsilon}\right)\left(N_{n}^{\varepsilon}+\delta_{n}^{\varepsilon}\right)\right)}-\frac{\sigma^{2}\left(\hat{y}_{n}^{\varepsilon}\right) N_{n}^{\varepsilon}}{2\left(e^{\rho t_{n}^{\varepsilon}}-f\left(\hat{y}_{n}^{\varepsilon}\right) N_{n}^{\varepsilon}\right)} .
\end{aligned}
$$


It remains to apply Proposition 2.10 together with 2.19 for $n$ large enough and $\varepsilon$ small enough to obtain

$$
\begin{aligned}
& \rho\left(\hat{U}\left(t_{n}^{\varepsilon}, x_{n}^{\varepsilon}\right)-\hat{V}_{\lambda}\left(t_{n}^{\varepsilon}, y_{n}^{\varepsilon}\right)\right) \\
& \leq \frac{\sigma^{2}\left(\hat{x}_{n}^{\varepsilon}\right) N_{n}^{\varepsilon}}{2\left(e^{\rho t_{n}^{\varepsilon}}-f\left(\hat{x}_{n}^{\varepsilon}\right) N_{n}^{\varepsilon}\right)}-\frac{\sigma^{2}\left(\hat{y}_{n}^{\varepsilon}\right) N_{n}^{\varepsilon}}{2\left(e^{\rho t_{n}^{\varepsilon}}-f\left(\hat{y}_{n}^{\varepsilon}\right) N_{n}^{\varepsilon}\right)}+O_{n}^{\varepsilon}(1) \\
& \leq L\left|\hat{x}_{n}^{\varepsilon}-\hat{y}_{n}^{\varepsilon}\right|+O_{n}^{\varepsilon}(1)
\end{aligned}
$$

for some $L>0$ and where $O_{n}^{\varepsilon}(1) \rightarrow 0$ as $n \rightarrow \infty$ and then $\varepsilon \rightarrow 0$. By continuity and 2.17) combined with Remark 2.3 and $(2.20)$, this contradicts 2.16$)$ for $n$ large enough.

\subsection{Supersolution property for the weak formulation}

In this part, we provide a lower bound $\underline{\mathrm{v}}_{\bar{\gamma}}$ for $\mathrm{v}_{\bar{\gamma}}$ that is a supersolution of $(1.17)$. It is constructed by considering a weak formulation of the stochastic target problem (1.15) in the spirit of [8, Section 5]. Since our methodology is slightly different, we provide the main arguments.

On $C\left(\mathbb{R}_{+}\right)^{5}$, let us now denote by $(\tilde{\zeta}:=(\tilde{a}, \tilde{b}, \tilde{\alpha}, \tilde{\beta}), \tilde{W})$ the coordinate process and let $\tilde{\mathbb{F}}^{\circ}=\left(\tilde{\mathcal{F}}_{s}^{\circ}\right)_{s \leq T}$ be its raw filtration. We say that a probability measure $\tilde{\mathbb{P}}$ belongs to $\tilde{\mathcal{A}}_{k}$ if $\tilde{W}$ is a $\tilde{\mathbb{P}}$-Brownian motion and if for all $0 \leq \delta \leq 1$ and $r \geq 0$ it holds $\tilde{\mathbb{P}}$-a.s. that

$$
\begin{gathered}
\tilde{a}=\tilde{a}_{0}+\int_{0}^{\cdot} \tilde{\beta}_{s} d s+\int_{0} \tilde{\alpha}_{s} d \tilde{W}_{s} \text { for some } \tilde{a}_{0} \in \mathbb{R}, \\
\sup _{\mathbb{R}_{+}}|\tilde{\zeta}| \leq k
\end{gathered}
$$

and

$$
\mathbb{E}^{\tilde{\mathbb{P}}}\left[\sup \left\{\left|\tilde{\zeta}_{s^{\prime}}-\tilde{\zeta}_{s}\right|, r \leq s \leq s^{\prime} \leq s+\delta\right\} \mid \tilde{\mathcal{F}}_{r}^{\circ}\right] \leq k \delta .
$$

For $\tilde{\phi}:=(y, \tilde{a}, \tilde{b}), y \in \mathbb{R}$, we define $\left(\tilde{X}^{x, \tilde{\phi}}, \tilde{Y}^{\tilde{\phi}}, \tilde{V}^{x, v, \tilde{\phi}}\right)$ as in $1.6-1.2-(1.7)$ associated to the control $(\tilde{a}, \tilde{b})$ with time- 0 initial condition $(x, y, v)$, and with $\tilde{W}$ in place of $W$. For $t \leq T$ and $k \geq 1$, we say that $\tilde{\mathbb{P}} \in \tilde{\mathcal{G}}_{k, \bar{\gamma}}(t, x, v, y)$ if

$$
\left[\tilde{V}_{T-t}^{x, v, \tilde{\phi}} \geq g\left(\tilde{X}_{T-t}^{x, \tilde{\phi}}\right) \text { and }-k \leq \gamma_{Y}^{\tilde{a}}\left(\tilde{X}^{x, \tilde{\phi}}\right) \leq \bar{\gamma}\left(\tilde{X}^{x, \tilde{\phi}}\right) \text { on } \mathbb{R}_{+}\right] \quad \tilde{\mathbb{P}}-\text { a.s. }
$$

We finally define

$$
\mathrm{v}_{\bar{\gamma}}^{k}(t, x):=\inf \left\{v=c+y x:(c, y) \in \mathbb{R} \times[-k, k] \text { s.t. } \tilde{\mathcal{A}}_{k} \cap \tilde{\mathcal{G}}_{k, \bar{\gamma}}(t, x, v, y) \neq \emptyset\right\},
$$


and

$$
\underline{\mathrm{v}}_{\bar{\gamma}}(t, x):=\liminf _{\substack{\left(k, t^{\prime}, x^{\prime}\right) \rightarrow(\infty, t, x) \\\left(t^{\prime}, x^{\prime}\right) \in[0, T) \times \mathbb{R}}} \mathrm{v}_{\bar{\gamma}}^{k}\left(t^{\prime}, x^{\prime}\right), \quad(t, x) \in[0, T] \times \mathbb{R} .
$$

The following is an immediate consequence of our definitions.

Proposition 2.12. $\mathrm{v}_{\bar{\gamma}} \geq \underline{\mathrm{v}}_{\bar{\gamma}}$ on $[0, T) \times \mathbb{R}$.

In the rest of this section, we show that $\underline{\mathrm{v}}_{\bar{\gamma}}$ is a viscosity supersolution of (1.17). We start with an easy remark.

Remark 2.13. Observe that the gamma constraint in $(2.24)$ implies that we can find $\varepsilon>0$ such that

$$
\frac{\varepsilon}{1+k \varepsilon^{-1}} \leq \sigma_{X}^{\tilde{a}}\left(\tilde{X}^{x, \tilde{\phi}}\right) \leq \varepsilon^{-1}+\varepsilon^{-2} \text { and }|\tilde{a}| \leq \varepsilon^{-1} \tilde{\mathbb{P}}-\text { a.s. }
$$

for all $\tilde{\mathbb{P}} \in \tilde{\mathcal{A}}_{k} \cap \tilde{\mathcal{G}}_{k, \bar{\gamma}}(t, x, v, y)$ and $k \geq 1$. Indeed, if $\tilde{a} \geq-\sigma / f$ then $-k \leq \gamma_{Y}^{\tilde{a}} \leq \bar{\gamma}$ implies

$$
\left(-\frac{k \sigma}{1+k f}\right) \vee\left(-\frac{\sigma}{f}\right) \leq \tilde{a} \leq \frac{\bar{\gamma} \sigma}{1-\bar{\gamma} f} \text { and } \tilde{a} f+\sigma \geq \sigma /(1+k f)
$$

Then our claim follows from (1.1)-(1.13). On the other hand, if $\sigma+\tilde{a} f<0$, then $\gamma_{Y}^{\tilde{a}} \leq \bar{\gamma}$ implies $\tilde{a} \geq \bar{\gamma} \sigma /(1-f \bar{\gamma}) \geq 0$, see $(1.13)$, while $\tilde{a}<-f / \sigma<0$, a contradiction.

We then show that $\underline{\underline{\gamma}} \frac{k}{\gamma}$ has linear growth, for $k$ large enough.

Proposition 2.14. There exists $k_{o} \geq 1$ such that $\left\{|\underline{\mathrm{v}} \underline{\mathrm{k}}|, k \geq k_{o}\right\}$ is uniformly bounded from above by a continuous map with linear growth.

Proof. a. First note that Remark 2.9 implies that $\left\{(\underline{\mathrm{v}} \bar{\gamma})^{+}, k \geq k_{o}\right\}$ is uniformly bounded from above by a map with linear growth, for some $k_{o}$ large enough.

b. Let us now fix $\tilde{\mathbb{P}} \in \tilde{\mathcal{A}}_{k} \cap \tilde{\mathcal{G}}_{k, \bar{\gamma}}(t, x, v, y)$. Using Remark 2.13 combined with 1.1) and the condition that $(\tilde{a}, \tilde{b}, \tilde{\alpha}, \tilde{\beta})$ is $\tilde{\mathbb{P}}$-essentially bounded, one can find $\check{\mathbb{P}} \sim \tilde{\mathbb{P}}$ under which $\int_{0}^{\cdot} \tilde{Y}_{s}^{\tilde{\phi}} d \tilde{X}_{s}^{x, \tilde{\phi}}$ is a martingale on $[0, T-t]$. Then, the condition $\tilde{V}_{T-t}^{x, v, \tilde{\phi}} \geq g\left(\tilde{X}_{T-t}^{x, \tilde{\phi}}\right) \tilde{\mathbb{P}}$-a.s. implies $v+\mathbb{E}^{\tilde{\mathbb{P}}}\left[\frac{1}{2} \int_{0}^{T-t} \tilde{a}_{s}^{2} f\left(\tilde{X}_{s}^{x, \tilde{\phi}}\right) d s\right] \geq \inf g>-\infty$, recall (1.16). By Remark 2.13 and (1.1), $v \geq \inf g-C>-\infty$, for some constant $C$ independent of $\tilde{\mathbb{P}} \in \cup_{k}\left(\mathcal{A}_{k} \cap \tilde{\mathcal{G}}_{k, \bar{\gamma}}(t, x, v, y)\right)$. Hence $\left\{(\underline{\mathrm{v}} \bar{k})^{-}, k \geq k_{o}\right\}$ is bounded by a constant.

We now prove that existence holds in the problem defining $\underline{\mathrm{v}} \frac{k}{\bar{\gamma}}$ and that it is lower-semicontinuous. 
Proposition 2.15. For all $(t, x) \in[0, T] \times \mathbb{R}$ and $k \geq 1$ large enough, there exists $(c, y) \in \mathbb{R} \times[-k, k]$ such that $\underline{\mathrm{v}} \bar{\gamma}(t, x)=c+y x$ and $\tilde{\mathcal{A}}_{k} \cap \tilde{\mathcal{G}}_{k, \bar{\gamma}}(t, c+x y, y) \neq \emptyset$. Moreover, $\underline{\mathrm{v}}_{\bar{\gamma}}^{k}$ is lower-semicontinuous for each $k \geq 1$ large enough.

Proof. By [20, Proposition XIII.1.5] and the condition (2.23) taken for $r=0$, the set $\tilde{\mathcal{A}}_{k}$ is weakly relatively compact. Moreover, [16, Theorem 7.10 and Theorem 8.1] implies that any limit point $\left(\mathbb{P}_{*}, t_{*}, x_{*}, c_{*}, y_{*}\right)$ of a sequence $\left(\mathbb{P}_{n}, t_{n}, x_{n}, c_{n}, y_{n}\right)_{n \geq 1}$ such that $\mathbb{P}_{n} \in \tilde{\mathcal{A}}_{k} \cap \tilde{\mathcal{G}}_{k, \bar{\gamma}}\left(t_{n}, x_{n}, c_{n}+x_{n} y_{n}, y_{n}\right)$ for each $n \geq 1$ satisfies $\mathbb{P}_{*} \in$ $\tilde{\mathcal{A}}_{k} \cap \tilde{\mathcal{G}}_{k, \bar{\gamma}}\left(t_{*}, x_{*}, c_{*}+x_{*} y_{*}, y_{*}\right)$. Since $\underline{\mathrm{v}} \frac{k}{\bar{\gamma}}$ is locally bounded, by Proposition 2.14 when $k \geq k_{o}$, the announced existence and lower-semicontinuity readily follow.

We can finally prove the main result of this section.

Theorem 2.16. The function $\underline{\mathrm{v}}_{\bar{\gamma}}$ is a viscosity supersolution of 1.17 . It has linear growth.

Proof. The linear growth property is an immediate consequence of the uniform linear growth of $\left\{|\underline{\mathrm{v}} \overline{\hat{\gamma}}|, k \geq k_{o}\right\}$ stated in Proposition 2.14. To prove the supersolution property, it suffices to show that it holds for each $\underline{\mathrm{v}} \overline{\mathrm{\gamma}}$, with $k \geq k_{o}$, and then to apply standard stability results, see e.g. [2].

a. We first prove the supersolution property on $[0, T) \times \mathbb{R}$. We adapt the arguments of [8] to our context. Let us consider a $C_{b}^{\infty}$ test function $\varphi$ and $\left(t_{0}, x_{0}\right) \in[0, T) \times \mathbb{R}$ such that

$$
\text { (strict) } \min _{[0, T) \times \mathbb{R}}\left(\underline{\mathrm{v}}_{\bar{\gamma}}^{k}-\varphi\right)=\left(\underline{\mathrm{v}}_{\bar{\gamma}}^{k}-\varphi\right)\left(t_{0}, x_{0}\right)=0 .
$$

Recall that $\underline{\mathrm{v}}_{\bar{\gamma}}^{k}$ is lower-semicontinuous by Proposition 2.15 .

Because the infimum is achieved in the definition of $\underline{\mathrm{v}} \bar{\gamma}$, by the afore-mentioned proposition, there exists $\left|y_{0}\right| \leq k$ and $\tilde{\mathbb{P}} \in \tilde{\mathcal{A}}_{k} \cap \tilde{\mathcal{G}}_{k}\left(t_{0}, x_{0}, v_{0}, y_{0}\right)$, such that $v_{0}:=$ $c_{0}+y_{0} x_{0}=\underline{\mathrm{v}} \overline{\bar{\gamma}}\left(t_{0}, x_{0}\right)$ for some $c_{0} \in \mathbb{R}$. Let us $\operatorname{set}(\tilde{X}, \tilde{Y}, \tilde{V}):=\left(\tilde{X}^{x_{0}, \tilde{\phi}}, \tilde{Y}^{\tilde{\phi}}, \tilde{V}^{x_{0}, v_{0}, \tilde{\phi}}\right)$ where $\tilde{\phi}=\left(y_{0}, \tilde{a}, \tilde{b}\right)$. Let $\theta_{o}$ be a stopping time for the augmentation of the raw filtration $\tilde{\mathbb{F}}^{\circ}$, and define

$$
\theta:=\theta_{o} \wedge \theta_{1} \text { with } \theta_{1}:=\inf \left\{s:\left|\tilde{X}_{s}-x_{0}\right| \geq 1\right\} .
$$

Then, it follows from Proposition 2.17 below that

$$
\tilde{V}_{\theta_{o}} \geq \underline{\mathrm{v}} \bar{k}\left(t_{0}+\theta_{o}, \tilde{X}_{\theta_{o}}\right) \geq \varphi\left(t_{0}+\theta_{o}, \tilde{X}_{\theta_{o}}\right),
$$

in which here and hereafter inequalities are taken in the $\tilde{\mathbb{P}}_{\text {-a.s. }}$ sense. After applying Itô's formula twice, the above inequality reads:

$$
\int_{0}^{\theta} \ell_{s} d s+\int_{0}^{\theta}\left(y_{0}-\partial_{x} \varphi\left(t_{0}, x_{0}\right)+\int_{0}^{s} m_{r} d r+\int_{0}^{s} n_{r} d \tilde{X}_{r}\right) d \tilde{X}_{s} \geq 0 .
$$


where

$$
\begin{gathered}
\ell:=\frac{1}{2} \tilde{a}^{2} f(\tilde{X})-\mathcal{L}^{\tilde{a}} \varphi\left(t_{0}+\cdot, \tilde{X} .\right), m:=\mu_{Y}^{\tilde{a}, \tilde{b}}(\tilde{X})-\mathcal{L}^{\tilde{a}} \partial_{x} \varphi\left(t_{0}+\cdot, \tilde{X} .\right) \\
n:=\gamma_{Y}^{\tilde{a}}(\tilde{X})-\partial_{x x}^{2} \varphi\left(t_{0}+\cdot, \tilde{X} .\right),
\end{gathered}
$$

with

$$
\mathcal{L}^{\tilde{a}}:=\partial_{t}+\frac{1}{2}\left(\sigma_{X}^{\tilde{a}}\right)^{2} \partial_{x x}^{2}
$$

For the rest of the proof, we recall $(2.22)$. Together with 1.1$)$ and Remark 2.13, this implies that $\sigma_{X}^{\tilde{a}}(\tilde{X}), \sigma_{X}^{\tilde{a}}(\tilde{X})^{-1}$ and $\mu_{X}^{\tilde{a}, \tilde{b}}(\tilde{X})$ are $\tilde{\mathbb{P}}$-essentially bounded. After performing an equivalent change of measure, we can thus find $\check{\mathbb{P}} \sim \tilde{\mathbb{P}}$ and a $\check{\mathbb{P}}_{\text {- }}$ Brownian motion $\check{W}$ such that:

$$
\tilde{X}=\int_{0}^{\cdot} \sigma_{X}^{\tilde{a}_{s}}\left(\tilde{X}_{s}\right) d \check{W}_{s}
$$

Clearly, both $\check{\mathbb{P}}$ and $\check{W}$ depend on $\left(\tilde{a}, \tilde{b}, y_{0}\right)$.

1. We first show that $y_{0}=\partial_{x} \varphi\left(t_{0}, x_{0}\right)$, and therefore

$$
\int_{0}^{\theta} \ell_{s} d s+\int_{0}^{\theta} \int_{0}^{s} m_{r} d r d \tilde{X}_{s}+\int_{0}^{\theta} \int_{0}^{s} n_{r} d \tilde{X}_{r} d \tilde{X}_{s} \geq 0 .
$$

Let $\check{\mathbb{P}}^{\lambda} \sim \check{\mathbb{P}}$ be the measure under which

$$
\check{W}^{\lambda}:=\check{W}+\int_{0} \lambda\left[\sigma_{X}^{\tilde{a}_{s}}\left(\tilde{X}_{s}\right)\right]^{-1}\left(y_{0}-\partial_{x} \varphi\left(t_{0}, x_{0}\right)\right) d s
$$

is a $\check{\mathbb{P}}^{\lambda}$-Brownian motion. Consider the case $\theta_{o}:=\eta>0$. Since all the coefficients are bounded, taking expectation under $\check{\mathbb{P}}^{\lambda}$ and using 2.26 imply

$$
\begin{aligned}
& C^{\prime} \eta \geq \lambda\left(y_{0}-\partial_{x} \varphi\left(t_{0}, x_{0}\right)\right)^{2} \mathbb{E}^{\check{\mathbb{P}}^{\lambda}}[\theta] \\
& +\mathbb{E}^{\check{\mathbb{P}}^{\lambda}}\left[\int_{0}^{\theta}\left(\int_{0}^{s} m_{r} d r+\int_{0}^{s} n_{r} d \tilde{X}_{r}\right) \lambda\left(y_{0}-\partial_{x} \varphi\left(t_{0}, x_{0}\right)\right) d s\right]
\end{aligned}
$$

for some $C^{\prime}>0$. We now divide both sides by $\eta$ and use the fact that $\left(\eta \wedge \theta_{1}\right) / \eta \rightarrow 1$ $\check{\mathbb{P}}^{\lambda}$-a.s. as $\eta \rightarrow 0$ to obtain

$$
C^{\prime} \geq \lambda\left(y_{0}-\partial_{x} \varphi\left(t_{0}, x_{0}\right)\right)^{2}
$$

Then, we send $\lambda \rightarrow \infty$ to deduce that $y_{0}=\partial_{x} \varphi\left(t_{0}, x_{0}\right)$.

2. We now prove that

$$
\partial_{x x}^{2} \varphi\left(t_{0}, x_{0}\right) \leq \gamma_{Y}^{\tilde{a}_{0}}\left(x_{0}\right) \leq \bar{\gamma}\left(x_{0}\right)
$$


We first consider the time change

$$
h(t)=\inf \left\{r \geq 0: \int_{0}^{r}\left[\left(\sigma_{X}^{\tilde{a}_{S}}\left(\tilde{X}_{s}\right)\right)^{2} \mathbf{1}_{[0, \theta]}(s)+\mathbf{1}_{[0, \theta] c}(s)\right] d s \geq t\right\} .
$$

Again, $\sigma_{X}^{\tilde{a}}(\tilde{X})$ and $\sigma_{X}^{\tilde{a}}(\tilde{X})^{-1}$ are essentially bounded by Remark 2.13 , so that $h$ is absolutely continuous and its density $\mathfrak{h}$ satisfies

$$
0<\underline{\mathfrak{h}} t \leq \mathfrak{h}(t):=\left[\left(\sigma_{X}^{\tilde{a}}(\tilde{X})\right)^{2} \mathbf{1}_{[0, \theta]}(t)+\mathbf{1}_{[0, \theta]}(t)\right]^{-1} \leq \overline{\mathfrak{h}} t
$$

for some constants $\mathfrak{h}$ and $\overline{\mathfrak{h}}$, for all $t \geq 0$. Moreover, $\hat{W}:=\tilde{X}_{h}$ is a Brownian motion in the time changed filtration. Let us now take $\theta_{o}:=h^{-1}(\eta)$ for some $0<\eta<1$. Then, 2.28) reads

$$
\begin{aligned}
0 \leq & \int_{0}^{\eta \wedge h^{-1}\left(\theta_{1}\right)} \ell_{h(s)} \mathfrak{h}(s) d s+\int_{0}^{\eta \wedge h^{-1}\left(\theta_{1}\right)} \int_{0}^{s} m_{h(r)} \mathfrak{h}(r) d r d \hat{W}_{s} \\
& +\int_{0}^{\eta \wedge h^{-1}\left(\theta_{1}\right)} \int_{0}^{s} n_{h(r)} d \hat{W}_{r} d \hat{W}_{s} .
\end{aligned}
$$

Since all the involved processes are continuous and bounded, and since $\left(\eta \wedge h^{-1}\left(\theta_{1}\right)\right) / \eta \rightarrow$ 1 a.s. as $\eta \rightarrow 0$, the above combined with [8, Theorem A.1 b. and Proposition A.3] implies that

$$
\gamma_{Y}^{\tilde{a}_{0}}\left(x_{0}\right)-\partial_{x x}^{2} \varphi\left(t_{0}, x_{0}\right)=\lim _{r \downarrow 0} n_{h(r)}=\lim _{r \downarrow 0} n_{r} \geq 0 .
$$

Since $\gamma_{Y}^{\tilde{a}}(\tilde{X}) \leq \bar{\gamma}(\tilde{X})$, this proves 2.29 .

3. It remains to show that the first term in the definition of $F[\varphi]\left(t_{0}, x_{0}\right)$ is also non-negative, recall (1.14). Again, let us take $\theta_{o}:=h^{-1}(\eta)$ and recall from 2. that $\lim _{\eta \rightarrow 0}\left(\eta \wedge h^{-1}\left(\theta_{1}\right)\right) / \eta=1 \check{\mathbb{P}}$-a.s. Note that $\tilde{a}$ being of the form 2.21) with the condition (2.22), it satisfies [8, Condition (A.2)], and so does $n$. Using [8, Theorem A.2 and Proposition A.3] and 2.31, we then deduce that $\ell_{0} \mathfrak{h}(0)-\frac{1}{2} n_{0} \geq 0$. Hence, 2.30 and direct computations based on 1.8 imply

$$
\begin{aligned}
0 & \leq \frac{1}{2} \tilde{a}_{0}^{2} f\left(x_{0}\right)-\mathcal{L}^{\tilde{a}_{0}} \varphi\left(t_{0}, x_{0}\right)-\frac{1}{2}\left(\gamma_{Y}^{\tilde{a}_{0}}\left(x_{0}\right)-\partial_{x x}^{2} \varphi\left(t_{0}, x_{0}\right)\right)\left(\sigma_{X}^{\tilde{a}_{0}}\left(x_{0}\right)\right)^{2} \\
& =\frac{1}{2} \tilde{a}_{0}^{2} f\left(x_{0}\right)-\partial_{t} \varphi\left(t_{0}, x_{0}\right)-\frac{1}{2} \gamma_{Y}^{\tilde{a}_{0}}\left(x_{0}\right)\left(\sigma_{X}^{\tilde{a}_{0}}\left(x_{0}\right)\right)^{2} \\
& =-\partial_{t} \varphi\left(t_{0}, x_{0}\right)-\frac{1}{2} \frac{\sigma^{2}\left(x_{0}\right)}{1-f\left(x_{0}\right) \gamma_{Y}^{\tilde{a}_{0}}\left(x_{0}\right)} \gamma_{Y}^{\tilde{a}_{0}}\left(x_{0}\right) \\
& \leq-\partial_{t} \varphi\left(t_{0}, x_{0}\right)-\frac{1}{2} \frac{\sigma^{2}\left(x_{0}\right)}{1-f\left(x_{0}\right) \partial_{x x}^{2} \varphi\left(t_{0}, x_{0}\right)} \partial_{x x}^{2} \varphi\left(t_{0}, x_{0}\right),
\end{aligned}
$$


in which we use the facts that $\partial_{x x}^{2} \varphi\left(t_{0}, x_{0}\right) \leq \gamma_{Y}^{\tilde{a}_{0}}\left(x_{0}\right) \leq \bar{\gamma}\left(x_{0}\right)$ and $z \mapsto z /(1-$ $\left.f\left(x_{0}\right) z\right)$ in non-decreasing on $\left(-\infty, \bar{\gamma}\left(x_{0}\right)\right] \subset\left(-\infty, 1 / f\left(x_{0}\right)\right)$, for the last inequality. b. We now consider the boundary condition at $T$. Since $\underline{\mathrm{v}} \overline{\mathrm{\gamma}}$ is a supersolution of $\bar{\gamma}-\partial_{x x}^{2} \varphi \geq 0$ on $[0, T) \times \mathbb{R}$, the same arguments as in [11, Lemma 5.1] imply that $\underline{\mathrm{v}} \overline{\bar{\gamma}}-\bar{\Gamma}$ is concave for any twice differentiable function $\bar{\Gamma}$ such that $\partial_{x x}^{2} \bar{\Gamma}=\bar{\gamma}$. The function $\underline{\mathrm{v}} \bar{\gamma}$ being lower-semicontinuous, the map

$$
x \mapsto G(x):=\liminf _{\substack{t^{\prime} \rightarrow T, x^{\prime} \rightarrow x \\ t^{\prime}<T}} \underline{\mathrm{v}} \frac{k}{\gamma}\left(t^{\prime}, x^{\prime}\right)
$$

is such that $G \geq g$ and $G-\bar{\Gamma}$ is concave. Hence, $G=(G-\bar{\Gamma})^{\text {conc }}+\bar{\Gamma} \geq(g-\bar{\Gamma})^{\text {conc }}+\bar{\Gamma}$ $=\hat{g}$.

It remains to state the dynamic programming principle used in the above proof.

Proposition 2.17. Fix $(t, x, v, y) \in[0, T] \times \mathbb{R}^{2} \times[-k, k]$ and let $\theta$ be a stopping time for the $\tilde{\mathbb{P}}$-augmentation of $\tilde{\mathbb{F}}^{\circ}$ that takes $\tilde{\mathbb{P}}$-a.s. values in $[0, T-t]$. Assume that $\tilde{\mathbb{P}} \in \tilde{\mathcal{A}}_{k} \cap \tilde{\mathcal{G}}_{k, \bar{\gamma}}(t, x, v, y)$. Then,

$$
\tilde{V}_{\theta}^{x, v, \tilde{\phi}} \geq \underline{\mathrm{v}} \overline{\bar{\gamma}}\left(t+\theta, \tilde{X}_{\theta}^{x, \tilde{\phi}}\right) \tilde{\mathbb{P}}-\text { a.s. },
$$

in which $\tilde{\phi}:=(y, \tilde{a}, \tilde{b})$.

Proof. Since $\underline{\underline{v}} \bar{k}$ is lower-semicontinuous and all the involved processes have continuous paths, up to approximating $\theta$ by a sequence of stopping times valued in finite time grids, it suffices to prove our claim in the case $\theta \equiv r \in[0, T-t]$. Let $\tilde{\mathbb{P}}_{\omega}$ be a regular conditional probability given $\tilde{\mathcal{F}}_{r}^{\circ}$ for $\tilde{\mathbb{P}}$. It coincides with $\tilde{\mathbb{P}}_{r}\left[\cdot \tilde{\mathcal{F}}_{r}^{\circ}\right](\omega)$ outside a set $N$ of $\tilde{\mathbb{P}}$-measure zero. Then, for all $\omega \notin N, 0 \leq \delta \leq 1$ and $r \geq 0$ the conditions 2.21)-(2.22)- 2.23 hold for $\tilde{\mathbb{P}}_{\omega}^{r}$ defined on $C\left(\mathbb{R}_{+}\right)^{5}$ by

$$
\tilde{\mathbb{P}}_{\omega}^{r}\left[\omega^{\prime} \in A\right]=\tilde{\mathbb{P}}_{\omega}\left[\omega_{r+}^{\prime} \in A\right] .
$$

Moreover, [9, Theorem 3.3] ensures that, after possibly modifying $N$,

$$
\begin{aligned}
& \tilde{\mathbb{P}}_{\omega}^{r}\left[\tilde{V}_{T-(t+r)}^{\xi_{r}(\omega), \vartheta_{r}(\omega), \hat{\phi}(\omega)} \geq g\left(\tilde{X}_{T-(t+r)}^{\xi_{r}(\omega), \hat{\phi}(\omega)}\right)\right]=1 \\
& \text { and } \tilde{\mathbb{P}}_{\omega}^{r}\left[\gamma_{Y}^{\tilde{a}}\left(\tilde{X}^{\xi_{r}(\omega), \hat{\phi}(\omega)}\right) \leq \bar{\gamma}\left(\tilde{X}^{\xi_{r}(\omega), \hat{\phi}(\omega)}\right) \text { on } \mathbb{R}_{+}\right]=1,
\end{aligned}
$$

for $\omega \notin N$, in which

$$
\left(\xi_{r}, \vartheta_{r}, \hat{\phi}\right):=\left(\tilde{X}_{r}^{x, \tilde{\phi}}, \tilde{V}_{r}^{x, v, \tilde{\phi}},\left(\tilde{Y}_{r}^{x, \tilde{\phi}}, \tilde{a}, \tilde{b}\right)\right) .
$$

This shows that $\vartheta_{r}(\omega) \geq \underline{\mathrm{v}} \bar{k}\left(t+r, \xi_{r}(\omega)\right)$ outside the null set $N$, which is the required result. 


\subsection{Conclusion of the proof and construction of almost optimal strategies}

We first conclude the proof of Theorem 1.4 .

Proof of Theorem 1.4. Proposition 2.5 and Theorem 2.8 imply that $\overline{\mathrm{v}}_{\bar{\gamma}} \geq \mathrm{v} \bar{\gamma}$ in which $\overline{\mathrm{v}}_{\bar{\gamma}}$ has linear growth and is a continuous viscosity solution of (1.17). On the other hand, Proposition 2.12 and Theorem 2.16 imply that $\underline{\mathrm{v}}_{\bar{\gamma}} \leq \mathrm{v}_{\bar{\gamma}}$ on $[0, T) \times \mathbb{R}$ in which $\underline{\mathrm{v}}_{\bar{\gamma}}$ has linear growth and is a viscosity supersolution of (1.17). By the comparison result of Theorem 2.11 applied with $\epsilon=0, \underline{\mathrm{v}}_{\bar{\gamma}} \geq \overline{\mathrm{v}}_{\bar{\gamma}}$. Hence,

$$
\mathrm{v}_{\bar{\gamma}}=\underline{\mathrm{v}}_{\bar{\gamma}}=\overline{\mathrm{v}}_{\bar{\gamma}} \text { on }[0, T) \times \mathbb{R} \text { and } \underline{\mathrm{v}}_{\bar{\gamma}}=\overline{\mathrm{v}}_{\bar{\gamma}} \text { on }[0, T] \times \mathbb{R}
$$

Since $\overline{\mathrm{v}}_{\bar{\gamma}}$ is continuous, this shows that

$$
\lim _{\substack{\left(t^{\prime}, x^{\prime}\right) \rightarrow(T, x) \\ t^{\prime}<T}} \mathrm{v} \bar{\gamma}\left(t^{\prime}, x^{\prime}\right)=\overline{\mathrm{v}}_{\bar{\gamma}}(T, x)=\underline{\mathrm{v}}_{\bar{\gamma}}(T, x) .
$$

Hence, $\mathrm{v}_{\bar{\gamma}}$ is a viscosity solution of $(1.17)$, with linear growth.

Remark 2.18 (Almost optimal controls). In the proof of Theorem 2.8, we have constructed a super-hedging strategy starting from $\overline{\mathrm{v}}_{\bar{\gamma}}^{\epsilon, K, \delta}(t, x)$. Since $\overline{\mathrm{v}_{\bar{\gamma}}^{\epsilon, K}, \delta}(t, x) \rightarrow$ $\overline{\mathrm{v}}_{\bar{\gamma}}(t, x)=\mathrm{v}_{\bar{\gamma}}(t, x)$ as $\delta, \epsilon \rightarrow 0$ and $K \rightarrow \infty$, this provides a way to construct super-hedging strategies associated to any initial wealth $v>\mathrm{v} \bar{\gamma}(t, x)$.

\section{Adding a resilience effect}

In this section, we explain how a resilience effect can be added to our model. In the discrete rebalancement setting, we replace the dynamics (1.4) by

$$
X^{n}=X_{0}+\int_{0}^{\cdot} \mu\left(X_{s}^{n}\right) d s+\int_{0}^{\cdot} \sigma\left(X_{s}^{n}\right) d W_{s}+R^{n},
$$

in which $R^{n}$ is defined by

$$
R^{n}=R_{0}+\sum_{i=1}^{n} \mathbf{1}_{\left[t_{i}^{n}, T\right]} \delta_{t_{i}^{n}}^{n} f\left(X_{t_{i}^{n}-}^{n}\right)-\int_{0}^{\cdot} \rho R_{s}^{n} d s
$$

for some $\rho>0$ and $R_{0} \in \mathbb{R}$. The process $R^{n}$ models the impact of past trades on the price, the last term in its dynamics is the resilience effect. Then, the continuous 
time dynamics becomes

$$
\begin{aligned}
X & =X_{0}+\int_{0}^{\cdot} \sigma\left(X_{s}\right) d W_{s}+\int_{0}^{\cdot} f\left(X_{s}\right) d Y_{s}+\int_{0}^{\cdot}\left(\mu\left(X_{s}\right)+a_{s}\left(\sigma f^{\prime}\right)\left(X_{s}\right)-\rho R_{s}\right) d s \\
R & =R_{0}+\int_{0}^{\cdot} f\left(X_{s}\right) d Y_{s}+\int_{0}^{\cdot}\left(a_{s}\left(\sigma f^{\prime}\right)\left(X_{s}\right)-\rho R_{s}\right) d s \\
V & =V_{0}+\int_{0}^{\cdot} Y_{s} d X_{s}+\frac{1}{2} \int_{0}^{\cdot} a_{s}^{2} f\left(X_{s}\right) d s .
\end{aligned}
$$

This is obtained as a straightforward extension of [4, Proposition 1.1].

Let $\mathrm{v}_{\bar{\gamma}}^{R}(t, x)$ be defined as the super-hedging price $\mathrm{v}_{\bar{\gamma}}(t, x)$ but for these new dynamics and for $R_{t}=0$. The following states that $\mathrm{v}_{\bar{\gamma}}^{R}=\mathrm{v}_{\bar{\gamma}}$, i.e. adding a resilience effect does not affect the super-hedging price.

Proposition 3.1. $\mathrm{v}_{\bar{\gamma}}=\mathrm{v}_{\bar{\gamma}}^{R}$ on $[0, T] \times \mathbb{R}$.

Proof. 1. To show that $\mathrm{v}_{\bar{\gamma}} \geq \mathrm{v}_{\bar{\gamma}}^{R}$, it suffices to reproduce the arguments of the proof of Theorem 2.8 in which the drift part of the dynamics of $X$ does not play any role. More precisely, these arguments show that $\overline{\mathrm{v}}_{\bar{\gamma}} \geq \mathrm{v} \frac{R}{\bar{\gamma}}$. Then, one uses the fact that $\mathrm{v}_{\bar{\gamma}}=\overline{\mathrm{v}}_{\bar{\gamma}}$, by 2.32 .

2. As for the opposite inequality, we use the weak formulation of Section 2.2 and a simple Girsanov's transformation. For ease of notations, we restrict to $t=0$. Fix $v>\mathrm{v}_{\bar{\gamma}}^{R}(0, x)$, for some $x \in \mathbb{R}$. Then, one can find $k \geq 1,(c, y) \in \mathbb{R} \times$ $[-k, k]$ satisfying $v=c+y x$, and $(a, b) \in \mathcal{A}_{k, \bar{\gamma}}(0, x)$ such that $V_{T} \geq g\left(X_{T}\right)$, with $(V, X, Y, R)$ defined by the corresponding initial data and controls. We let

$$
a=a_{0}+\int_{0} \beta_{s} d s+\int_{0} \alpha_{s} d W_{s}
$$

be the decomposition of $a$ into an Itô process, see Section 1.1. Let $\mathbb{Q}^{R} \sim \mathbb{P}$ be the probability measure under which $W^{R}:=W-\int_{0}^{\cdot}\left(\rho R_{s} / \sigma\left(X_{s}\right)\right) d s$ is a $\mathbb{Q}^{R}$-Brownian motion, recall (1.1). Then,

$$
\begin{aligned}
& X=X_{0}+\int_{0}^{\cdot} \sigma\left(X_{s}\right) d W_{s}^{R}+\int_{0}^{\cdot} f\left(X_{s}\right) d Y_{s}+\int_{0}^{\cdot}\left(\mu\left(X_{s}\right)+a_{s}\left(\sigma f^{\prime}\right)\left(X_{s}\right)\right) d s \\
& Y=Y_{0}+\int_{0}^{\cdot}\left(b_{s}+a_{s} \rho R_{s} / \sigma\left(X_{s}\right)\right) d s+\int_{0} a_{s} d W_{s}^{R} \\
& a=a_{0}+\int_{0}^{\cdot}\left(\beta_{s}+\alpha_{s} \rho R_{s} / \sigma\left(X_{s}\right)\right) d s+\int_{0} \alpha_{s} d W_{s}^{R} \\
& V=V_{0}+\int_{0}^{\cdot} Y_{s} d X_{s}+\frac{1}{2} \int_{0} a_{s}^{2} f\left(X_{s}\right) d s .
\end{aligned}
$$


Upon seeing $\left(a, b+a \rho R / \sigma(X), \alpha, \beta+\alpha \rho R / \sigma(X), W^{R}\right)$ as a generic element of the canonical space $C([0, T])^{5}$ introduced in Section 2.2 , then $\mathbb{Q}^{R}$ belongs to $\tilde{\mathcal{A}}_{k} \cap$ $\tilde{\mathcal{G}}_{k, \bar{\gamma}}(t, x, v, y)$, and therefore $v>\underline{\mathrm{v}}_{\bar{\gamma}}(0, x)$. Hence, $\mathrm{v}_{\bar{\gamma}}^{R}(0, x) \geq \mathrm{v}_{\bar{\gamma}}(0, x)$, and thus $\mathrm{v}_{\bar{\gamma}}^{R}(0, x) \geq \mathrm{v}_{\bar{\gamma}}(0, x)$ by 2.32 .

\section{Numerical approximation and examples}

In this section, we provide an example of numerical schemes that converges towards the unique continuous viscosity solution of (1.17) with linear growth. The scheme is then exemplified using two numerical applications in the case of constant market impact and gamma constraint.

\subsection{Finite difference scheme}

Given a map $\phi$ and $h:=\left(h_{t}, h_{x}\right) \in(0,1)^{2}$, define

$$
\begin{aligned}
& L_{1}^{h}(t, x, y, \phi):=-\frac{\phi\left(t+h_{t}, x\right)-y}{h_{t}}-\frac{\sigma^{2}(x) G^{h}(t, x, y, \phi)}{2\left(1-f(x) G^{h}(t, x, y, \phi)\right)} \\
& L_{2}^{h}(t, x, y, \phi):=\bar{\gamma}(x)-G^{h}(t, x, y, \phi)
\end{aligned}
$$

where

$$
G^{h}(t, x, y, \phi):=\frac{\phi\left(t+h_{t}, x+h_{x}\right)+\phi\left(t+h_{t}, x-h_{x}\right)-2 y}{h_{x}^{2}} .
$$

The numerical scheme is set on the grid $\pi_{h}:=\left\{\left(t_{i}, x_{j}\right)=\left(i h_{t}, \underline{x}+j h_{x}\right): i \leq\right.$ $\left.n_{t}, j \leq n_{x}\right\}$, with $n_{t} h_{t}=T$ for some $n_{t} \in \mathbb{N}$, and $n_{x} h_{x}=\bar{x}-\underline{x}$, for some real numbers $\underline{x}<\bar{x}$. To paraphrase, $\mathrm{v}_{\bar{\gamma}}^{h}$ is defined on $\pi_{h}$ as the solution of

$$
\begin{aligned}
S\left(h, t_{i}, x_{j}, \mathrm{v}_{\bar{\gamma}}^{h}\left(t_{i}, x_{j}\right), \mathrm{v}_{\bar{\gamma}}^{h}\right) & =0 \text { for } i<n_{t}, 1 \leq j \leq n_{x}-1 \\
\mathrm{v}_{\bar{\gamma}}^{h} & =\hat{g} \text { on } \pi_{h} \cap\{(\{T\} \times \mathbb{R}) \cup([0, T] \cap\{\underline{x}, \bar{x}\})\}
\end{aligned}
$$

where

$$
S(h, t, x, y, \phi):=(\bar{w}-y) \vee(y-\underline{w}) \wedge \min _{l=1,2}\left\{L_{l}^{h}(t, x, y, \phi)\right\}
$$

with $\bar{w}$ and $\underline{w}$ as in Remark 2.6 .

Theorem 4.1. The equation (4.1) admits a unique solution $\mathrm{v} \frac{h}{\gamma}$, for all $h:=$ $\left(h_{t}, h_{x}\right) \in(0,1)^{2}$. Moreover, if $h_{t} / h_{x}^{2} \rightarrow 0$ and $h_{x}^{2} \rightarrow 0$, then $\mathrm{v}_{\bar{\gamma}}^{h}$ converges locally uniformly to the unique continuous viscosity solution of (1.17) that has linear growth. 
Proof. The existence of a solution, that is bounded by the map with linear growth $|\bar{w}|+|\underline{w}|$, is obvious. We now prove uniqueness. First observe that $L_{2}^{h}$ is strictly increasing in its $y$-component, and that

$$
\frac{\partial L_{1}^{h}}{\partial y}(t, x, y, \phi)=\frac{1}{h_{t}}+\frac{\sigma^{2}(x)}{h_{x}^{2}\left(1-f(x) G^{h}(t, x, y, \phi)\right)^{2}}>0
$$

on the domain $\left\{y: L_{2}^{h}\left(t_{i}, x_{j}, y, \phi\right) \geq 0\right\}$. Uniqueness of the solution follows.

It is easy to see that $\phi \mapsto S(\cdot, \phi)$ is non-decreasing, so that our scheme is monotone. Consistency is clear. Moreover, it is not difficult to check that the comparison result of Theorem 2.11 extends to this equation (there is an equivalence of the notions of super- and subsolutions in the class of functions $w$ such that $\underline{w} \leq w \leq \bar{w})$. It then follows from [3, Theorem 2.1] that $\mathrm{v}_{\bar{\gamma}}^{h}$ converges locally uniformly to the unique continuous viscosity solution with linear growth of

$$
[(\bar{w}-\varphi) \vee(\varphi-\underline{w}) \wedge F[\varphi]] \mathbf{1}_{[0, T)}+(\varphi-\hat{g}) \mathbf{1}_{\{T\}}=0 .
$$

In view of (2.32), Remark 2.6 and Theorem $1.4, \mathrm{v}_{\bar{\gamma}}$ is the unique viscosity solution of the above equation.

\subsection{Numerical examples: the fixed impact case}

To illustrate the above numerical scheme, we place ourselves in the simpler case where $f \equiv \lambda>0$ and $\bar{\gamma}>0$ are constant. The dynamics of the stock is given by the Bachelier model

$$
d X_{t}=\sigma d W_{t},
$$

with $\sigma:=0.2$. In the following, $T=2$.

First, we consider a European Butterfly option with three strikes $K_{1}=-1<$ $K_{2}=0<K_{3}=1$, where $K_{1}+1 /(2 \bar{\gamma}) \leq K_{2} \leq K_{3}-1 /(2 \bar{\gamma})$. Its pay-off is

$$
g(x)=\left(x-K_{1}\right)^{+}-2\left(x-K_{2}\right)^{+}+\left(x-K_{3}\right)^{+},
$$

and the corresponding face-lifted function $\hat{g}$ can be computed explicitly:

$$
\begin{aligned}
\hat{g}(x)= & \frac{\bar{\gamma}}{2}\left(x-x_{1}^{-}\right)^{2} \mathbf{1}_{\left[x_{1}^{-}, x_{1}^{+}\right)}+\left(x-K_{1}\right) \mathbf{1}_{\left[x_{1}^{+}, K_{2}\right)} \\
& +\left(x-K_{1}-2\left(x-K_{2}\right)\right) \mathbf{1}_{\left[K_{2}, x_{2}^{-}\right)} \\
& +\left(\frac{\bar{\gamma}}{2}\left(x-x_{2}^{+}\right)^{2}+2 K_{2}-\left(K_{1}+K_{3}\right)\right) \mathbf{1}_{\left[x_{2}^{-}, x_{2}^{+}\right)} \\
& +\left(2 K_{2}-\left(K_{1}+K_{3}\right)\right) \mathbf{1}_{\left[x_{2}^{+},+\infty\right)},
\end{aligned}
$$

where $x_{1}^{ \pm}=K_{1} \pm 1 /(2 \bar{\gamma})$ and $x_{2}^{ \pm}=K_{3} \pm 1 /(2 \bar{\gamma})$. 
In Figure 1, we separately show the effect of the gamma constraint and of the market impact. As observed in Remark 1.9, the price is non-decreasing with respect to the impact parameter $\lambda$ and bounded from below by the hedging price obtained in the model without impact nor gamma constraint. On the left and right tails of the curves, we observe the effect of the gamma constraint. It does not operate around $x=0$ where the gamma is non-positive. The effect of the market impact operates only in areas of high convexity (around $x=-1.5$ and $x=1.5$ ) or of high concavity (around $x=0$ ).
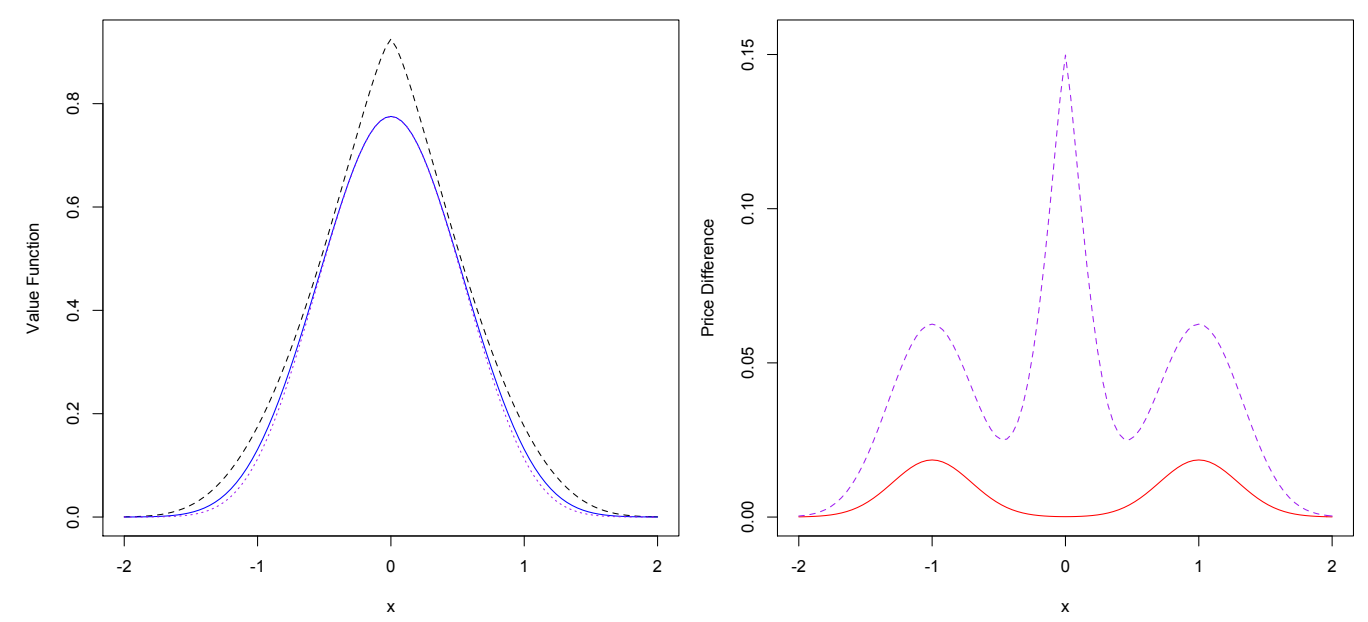

Figure 1: Left: Super-hedging price of the Butterfly option. Dashed line: $\lambda=0.5, \bar{\gamma}=1.75$; solid line: $\lambda=0, \bar{\gamma}=1.75$; dotted line: $\lambda=0, \bar{\gamma}=+\infty$. Right: Difference with the price associated to $\lambda=0, \bar{\gamma}=+\infty$. Dashed line: $\lambda=0.5, \bar{\gamma}=1.75 ;$ solid line: $\lambda=0, \bar{\gamma}=1.75$.

In Figure 2, we perform similar computations but for a call spread option, where

$$
g(x)=\left(x-K_{1}\right)^{+}-\left(x-K_{2}\right)^{+},
$$

with $K_{1}=-1<K_{2}=1$ such that $K_{1}+1 /(2 \bar{\gamma}) \leq K_{2}$. The face-lifted function $\hat{g}$ is given by

$$
\hat{g}(x)=\frac{\bar{\gamma}}{2}\left(x-x^{-}\right)^{2} \mathbf{1}_{\left[x^{-}, x^{+}\right)}+\left(x-K_{1}\right) \mathbf{1}_{\left[x^{+}, K_{2}\right)}+\left(K_{2}-K_{1}\right) \mathbf{1}_{\left[K_{2},+\infty\right)}
$$

with $x^{ \pm}=K_{1} \pm 1 /(2 \bar{\gamma})$. 

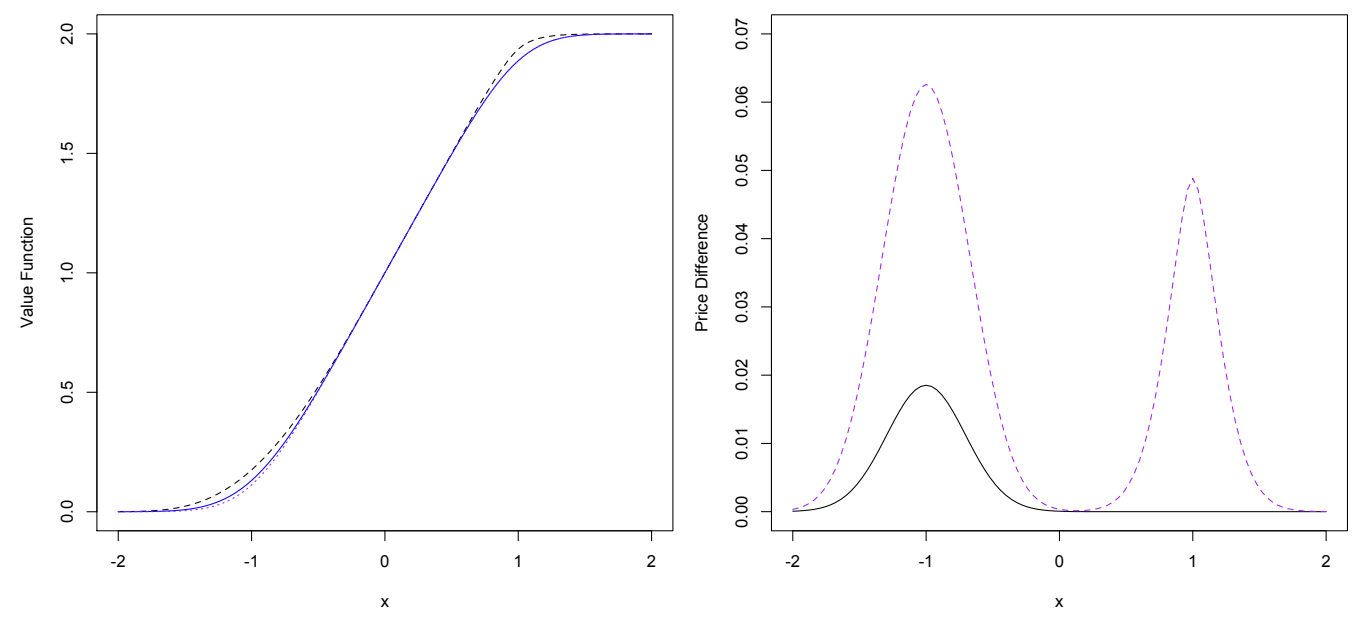

Figure 2: Left: Super-hedging price of the Call Spread option. Dashed line: $\lambda=0.5, \bar{\gamma}=1.75$; solid line: $\lambda=0, \bar{\gamma}=1.75$; dotted line: $\lambda=0, \bar{\gamma}=+\infty$. Right: Difference with the price associated to $\lambda=0, \bar{\gamma}=+\infty$. Dashed line: $\lambda=0.5, \bar{\gamma}=1.75$; solid line: $\lambda=0, \bar{\gamma}=1.75$.

\section{Appendix}

The following is very standard, we prove it for completeness.

Lemma 5.1. A upper-semicontinuous (resp. lower-semicontinuous) map is a viscosity subsolution (resp. supersolution) of

$$
F_{\kappa}^{\epsilon}[\varphi] \mathbf{1}_{[0, T)}+\left(\varphi-\hat{g}_{K}^{\epsilon}\right) \mathbf{1}_{\{T\}}=0
$$

if and only if it is a viscosity subsolution (resp. supersolution) of $F_{\kappa,-}^{\epsilon, K}[\varphi]=0$ (resp. $\left.F_{\kappa,+}^{\epsilon, K}[\varphi]=0\right)$.

Proof. The equivalence on $[0, T)$ is evident, we only consider the parabolic boundary $\{T\} \times \mathbb{R}$. Since $F_{\kappa,+}^{\epsilon, K} \geq F_{\kappa}^{\epsilon}$ and $F_{\kappa,-}^{\epsilon, K} \leq F_{\kappa}^{\epsilon}$, only one implication is not completely trivial.

a. Let $v$ be a viscosity supersolution of $F_{\kappa,+}^{\epsilon, K}[\varphi]=0$, and $\varphi \in C^{2}$ be a test function such that

$$
\text { (strict) } \min _{[0, T] \times \mathbb{R}}(v-\varphi)=(v-\varphi)\left(T, x_{0}\right)=0,
$$


for some $x_{0} \in \mathbb{R}$. We define a new test function $\phi \in C^{2}$,

$$
\phi(t, x):=\varphi(t, x)-C(T-t),
$$

so that $\partial_{t} \phi=\partial_{t} \varphi+C$. For $C>0$ large enough,

$$
\min _{x^{\prime} \in D_{\kappa}^{\epsilon}} \min \left\{-\partial_{t} \phi-\frac{\sigma^{2}\left(x^{\prime}\right) \partial_{x x} \phi}{2\left(1-f\left(x^{\prime}\right) \partial_{x x} \phi\right)}, \bar{\gamma}\left(x^{\prime}\right)-\partial_{x x} \phi\right\}<0
$$

at $\left(T, x_{0}\right)$. Since,

$$
\text { (strict) } \min _{[0, T] \times \mathbb{R}}(v-\phi)=(v-\phi)\left(T, x_{0}\right)=0,
$$

it must hold that $F_{\kappa,+}^{\epsilon, K}[\phi]\left(T, x_{0}\right) \geq 0$, and therefore

$$
v\left(T, x_{0}\right)-\hat{g}_{K}^{\epsilon}\left(x_{0}\right)=\varphi\left(T, x_{0}\right)-\hat{g}_{K}^{\epsilon}\left(x_{0}\right)=\phi\left(T, x_{0}\right)-\hat{g}_{K}^{\epsilon}\left(x_{0}\right) \geq 0 .
$$

b. Let now $v$ be a viscosity subsolution of $F_{\kappa,-}^{\epsilon, K}[\varphi]=0$, and $\varphi \in C^{2}$ be a test function such that

$$
\text { (strict) } \max _{[0, T] \times \mathbb{R}}(v-\varphi)=(u-\varphi)\left(T, x_{0}\right)
$$

for some $x_{0} \in \mathbb{R}$. Then, $F_{\kappa,-}^{\epsilon, K}[\varphi]\left(T, x_{0}\right) \leq 0$. By replacing $\varphi$ by $\phi$, defined for $\alpha>0$ as

$$
\phi(t, x):=\varphi\left(t, x_{0}+\alpha\left(x-x_{0}\right)\right)+C(T-t),
$$

we obtain a new test function at $\left(T, x_{0}\right)$. Since $\inf \bar{\gamma}>0$, recall 1.1 , we can take $\alpha$ small enough so that

$$
\min _{x^{\prime} \in D_{\kappa}^{\epsilon}}\left\{\bar{\gamma}\left(x^{\prime}\right)-\partial_{x x} \phi\left(T, x_{0}\right)\right\}>0
$$

As in the previous step, we can now choose $C>0$ such that

$$
\min _{x^{\prime} \in D_{\kappa}^{\epsilon}}\left\{-\partial_{t} \phi-\frac{\sigma^{2}\left(x^{\prime}\right) \partial_{x x} \phi}{2\left(1-f\left(x^{\prime}\right) \partial_{x x} \phi\right)}\right\}>0
$$

at $\left(T, x_{0}\right)$. Since $F_{\kappa,-}^{\epsilon, K}[\phi]\left(T, x_{0}\right) \leq 0$, we conclude that $v\left(T, x_{0}\right)=\phi\left(T, x_{0}\right) \leq$ $\hat{g}_{K}^{\epsilon}\left(x_{0}\right)$. 


\section{References}

[1] F. Abergel and G. Loeper. Pricing and hedging contingent claims with liquidity costs and market impact. SSRN.

[2] G. Barles. Solution de viscosités des équations d'Hamilton Jacobi, volume 17 of Mathématiques et Applications. Springer Verlag, 1994.

[3] G. Barles and P.E. Souganidis. Convergence of approximation schemes for fully nonlinear second order equations. In Decision and Control, 1990., Proceedings of the 29th IEEE Conference on, pages 2347-2349. IEEE, 1990.

[4] B. Bouchard, G. Loeper, and Y. Zou. Almost-sure hedging with permanent price impact. To appear in Finance and Stochastics, 2015.

[5] B. Bouchard and M. Nutz. Stochastic target games and dynamic programming via regularized viscosity solutions. To appear in Mathematics of Operation Research, 2013.

[6] U. Çetin, R. A. Jarrow, and P. Protter. Liquidity risk and arbitrage pricing theory. Finance Stoch., 8(3):311-341, 2004.

[7] U. Çetin, H. M. Soner, and N. Touzi. Option hedging for small investors under liquidity costs. Finance Stoch., 14(3):317-341, 2010.

[8] P. Cheridito, H. M. Soner, and N. Touzi. The multi-dimensional superreplication problem under gamma constraints. In Annales de l'IHP Analyse non linéaire, volume 22, pages 633-666, 2005.

[9] J. Claisse, D. Talay, and X. Tan. A note on solutions to controlled martingale problems and their conditioning. 2014.

[10] M. G. Crandall, H. Ishii, and P.-L. Lions. User's guide to viscosity solutions of second order partial differential equations. Bull. Amer. Math. Soc. (N.S.), 27(1):1-67, 1992.

[11] J. Cvitanić, H. Pham, and N. Touzi. Super-replication in stochastic volatility models under portfolio constraints. Journal of Applied Probability, 36(2), 523$545,1999$.

[12] R. Frey. Perfect option hedging for a large trader. Finance and Stochastics, 2:115-141, 1998.

[13] H. Ishii. On the equivalence of two notions of weak solutions, viscosity solutions and distribution solutions. Funkcial. Ekvac, 38(1):101-120, 1995. 
[14] R. Jensen. The maximum principle for viscosity solutions of fully nonlinear second order partial differential equations. Arch. Rational Mech. Anal., 101(1):1-27, 1988.

[15] N. V. Krylov. On the rate of convergence of finite-difference approximations for bellmans equations with variable coefficients. Probability theory and related fields, 117(1):1-16, 2000.

[16] T. G. Kurtz and P. E. Protter. Weak convergence of stochastic integrals and differential equations. In Probabilistic models for nonlinear partial differential equations, pages 1-41. Springer, 1996.

[17] H. Liu and J. M. Yong. Option pricing with an illiquid underlying asset market. Journal of Economic Dynamics and Control, 29:2125-2156, 2005.

[18] G. Loeper. Option Pricing with Market Impact and Non-Linear Black and Scholes PDEs. SSRN.

[19] G. Loeper. Solution of a fully non-linear Black and Scholes equation coming from a linear market impact model. SSRN.

[20] D. Revuz and M. Yor. Continuous martingales and Brownian motion, volume 293. Springer Science \& Business Media, 1999.

[21] P. J. Schönbucher and P. Wilmott. The feedback effects of hedging in illiquid markets. SIAM Journal on Applied Mathematics, 61:232-272.

[22] K. R. Sircar and G. Papanicolaou. Generalized black-scholes models accounting for increased market volatility from hedging strategies. Applied Mathematical Finance, 5(1):45-82, 1998.

[23] H. M. Soner and N. Touzi. Superreplication under gamma constraints. SIAM J. Control Optim., 39:73-96, 2000.

[24] H. M. Soner and N. Touzi. The dynamic programming equation for second order stochastic target problems. SIAM Journal on Control and Optimization, pages 2344-2365. 